\title{
Stochastic attractors for non-ergodic Markov processes: some examples
}

\author{
Paolo Dai Pra \\ Ida Minelli \\ Dipartimento di Matematica Pura e Applicata \\ Università di Padova \\ Via Belzoni 7, 35131 Padova, Italy \\ daipra@math.unipd.it \\ minelli@math.unipd.it
}

\begin{abstract}
We discuss the notion of stochastic attractor for Markov processes. We exhibit some non trivial examples of Markov processes possessing more than one stationary distributions, for which the stochastic attractor can be explicitly determined.
\end{abstract}

Keywords and phrases: Random Dynamical Systems, Markov processes.

AMS 2000 subject classification numbers: 60K35, 82C22.

\section{Introduction}

One of the most classical and relevant problems in the Theory of Markov processes is the analysis of the long-time behavior, in particular the relaxation to the possibly non unique stationary distributions. The usual setup is as follows. A (time homogeneous) Markov process is assigned in terms of a consistent family of transition probabilities $P_{t}(x, d y), t \geq 0$ on some measurable space $(X, X)$. In the case of processes in discretetime it suffices to give the "one-step" transition probability $P(x, d y):=P_{1}(x, d y)$, while, in the continuous-time case, transition probabilities may be given indirectly by assigning the associated semigroup $T_{t}, t \geq 0$, or its infinitesimal generator $L$. Once the space of probability measures on $(X, X)$ is provided with a topology, that may depend on additional structure on $X$ (metric, norm, ...), a fundamental problem is to determine the limit points of the sequence $\left(P_{t}(x, d y)\right)_{t \geq 0}$ as $t \rightarrow+\infty$, their dependence on $x$ and their relations with the set of stationary distributions. The most regular possible behavior is when there is a unique stationary distribution $\mu$, and $P_{t}(x, d y) \rightarrow \mu$ as $t \rightarrow+\infty$, independently of the choice of $x$. If this happens we 
say that the Markov process is ergodic. Ergodicity can then be refined by the rate at which the convergence $P_{t}(x, d y) \rightarrow \mu$ takes place.

In analogy with deterministic systems, an alternative view of the long-time behavior of a stochastic process should come from the search of (possibly random) subsets of $X$ that are invariant for the dynamics. For this to make sense, one first has to define the process in terms of a dynamical system affected by noise, and then define properly the notion of invariance. In discrete time, for instance, a Markov process $\left(x_{t}\right)_{t \geq 0}$, can be constructed by letting the transition between $x_{t}$ and $x_{t+1}$ be realized by first generating a random number $\omega_{t}$, uniformly distributed in $[0,1]$, and then by applying a deterministic rule to $x_{t}$ and $\omega_{t}$. In other words we are assuming dynamics to be given by a difference equation of the form

$$
x_{t+1}=f\left(x_{t}, \omega_{t}\right) .
$$

In more precise terms, we let $\Omega=[0,1]^{\mathbb{Z}}$, provided with the natural product $\sigma$-field $\mathcal{F}$, and with the probability $\mathbb{P}$ given by the infinite product of the Lebesgue measure on $[0,1]$. Note that the projections $\omega \mapsto \omega_{t}$, as random variables in $(\Omega, \mathcal{F}, \mathbb{P})$, are i.i.d., uniformly distributed in $[0,1]$. The function $f(\cdot, \cdot)$ is a measurable function which realizes the transition probability $P(x, d y)$, i.e., for $A \in X, x \in X, t \in \mathbb{Z}$,

$$
\mathbb{P}\left(\left\{\omega \in \Omega: f\left(x, \omega_{t}\right) \in A\right\}\right)=P(x, A) .
$$

It can be shown that this model is very general: every transition probability satisfying some mild conditions (see $[\mathrm{K}]$ ) can be realized as above. Having defined the one step transition rule (1.1), we can obtain more generally the $t$-steps transition rules

$$
\varphi(t, \omega): X \rightarrow X
$$

that are recursively defined as follows:

$$
\begin{aligned}
\varphi(0, \omega) x & \equiv x \\
\varphi(t+1, \omega) x & =f\left(\varphi(t, \omega) x, \omega_{t}\right) .
\end{aligned}
$$

Clearly, $\varphi(t, \omega) x$ depends on $\omega$ only through $\omega_{0}, \omega_{1}, \ldots, \omega_{t-1}$. Moreover, by independence of the $\omega_{t}$ 's, $(\varphi(t, \omega) x)_{t \geq 0}$ is a Markov process, and $\varphi(t, \omega) x$ has distribution $P_{t}(x, d y)$.

In what follows we want to allow the initial time to be an arbitrary integer time $s$. To do so we define the shift maps $\left(\theta_{t}\right)_{t \in \mathbb{Z}}$ on $\Omega$ by

$$
\left(\theta_{t} \omega\right)_{t^{\prime}}=\omega_{t+t^{\prime}}
$$

Note that the $\theta_{t}$ 's are $\mathbb{P}$-preserving maps. Now, for $x \in X, s \in \mathbb{Z}$ and $t \in \mathbb{Z}^{+}$, $\varphi\left(t, \theta_{s} \omega\right) x$ has to be interpreted as the state at time $t+s$ of the stochastic system that started at $x$ at time $s$. The maps $\varphi(t, \omega), t \in \mathbb{Z}^{+}, \omega \in \Omega$, satisfy the semigroup or cocycle property

$$
\varphi(t+s, \omega)=\varphi\left(t, \theta_{s} \omega\right) \circ \varphi(s, \omega),
$$


for every $s, t \in \mathbb{Z}^{+}$and $\omega \in \Omega$.

In order to include more general realizations as well as the continuous time case, we are led to the following abstract setting. We aim at realizing a consistent family of transition probabilities $P_{t}(x, d y), t \in \mathbb{T}^{+}, \mathbb{T}=\mathbb{Z}$ or $\mathbb{R}$, in terms of a stochastic flow, given by assigning a probability space $(\Omega, \mathcal{F}, \mathbb{P})$, a family $\left(\theta_{t}\right)_{t \in \mathbb{T}}$ of $\mathbb{P}$-preserving maps, and a family of measurable maps $\varphi(t, \omega)$ satisfying (1.3) for $s, t \in \mathbb{T}^{+}$. This flow, that will be defined more precisely in Section 2, is called a random dynamical system.

We remark at this point that with this construction we have realized on the same probability space a family of Markov processes with the same transition probability indexed by the initial condition $x$. This is an example of what is often referred to as coupling, that is a widely used tool in stochastic processes (see [Li1, Li2, FG] for a systematic treatment). There is by no means a unique way of realizing a coupling: the choice of the "right" coupling depends on the purposes.

Now we go back to the problem of describing the long-time behavior of a stochastic system in terms on invariant sets. In general the forward paths $t \mapsto \varphi(t, \omega) x$ have no interesting long-time behavior in the almost sure sense (or in probability), as it is seen in the simple example $f\left(x, \omega_{t}\right) \equiv \omega_{t}$. Both in the context of Markov Chain Monte Carlo simulation ([Br, PW, Fi] $)$ and in the general theory of random dynamical systems $([\mathrm{A}, \mathrm{Ch}, \mathrm{C}, \mathrm{CF}])$ it has been shown that a successful approach is the so-called pull-back approach, which consists in starting the system at a large negative time $-t$ and observe it at the fixed time 0 rather than starting at time 0 and observing it at a large time $t$. The idea is that while in the more familiar "forward" view one keeps seeing random dynamical fluctuations, the pull-back provides a sort of "photography" of how the system looks like after a long aging. Therefore we consider the pull-back trajectories, defined by $t \mapsto \varphi\left(t, \theta_{-t} \omega\right) x$. An essentially simple but relevant property of pull-back trajectories can be seen when the flow $\varphi(t, \omega)$ models a irreducible (and aperiodic, if in discrete-time) Markov chain with finite state space. Under the condition

$$
T(\omega):=\min \left\{t>0: \forall x, y \in X \varphi\left(t, \theta_{-t} \omega\right) x=\varphi\left(t, \theta_{-t} \omega\right) y\right\}<+\infty \text { a.s. }
$$

that can be satisfied by an appropriate choice of the flow, we have

$$
\varphi\left(t, \theta_{-t} \omega\right) x=x_{\infty}(\omega):=\varphi\left(T(\omega), \theta_{-T(\omega)} \omega\right) x
$$

for every $t \geq T(\omega)$, and $x_{\infty}(\omega)$ is independent of the initial state $x$. Moreover, $x_{\infty}(\omega)$ is distributed according to the unique stationary distribution $\mu$, and it is invariant for the dynamics in the following sense: for each $\omega$ and $t \geq 0$, we have $\varphi(t, \omega) x_{\infty}(\omega)=x_{\infty}\left(\theta_{t} \omega\right)$. Thus, perfect sampling from $\mu$ is realized in finite (random) time. In particular, this shows that the sequence $\left(\varphi\left(t, \theta_{-t} \omega\right) x\right)_{t \geq 0}$ converges a.s. to a limit independent of $x$. This is the celebrated coupling from the past algorithm of Propp \& Wilson ([PW]).

For more general systems, such as non-ergodic Markov processes with infinite state space, the aim is to prove that the pull-back trajectories are attracted, in a suitable sense, by a dynamically invariant random subset of the state space, that is called random attractor. Although existence and various qualitative features of stochastic 
attractors are known (see e.g. $[\mathrm{CF}]$ ), non-trivial examples of explicit computation of the attractor for processes possessing more than one stationary distribution, to our knowledge, are less encountered in the literature (with the exception of some important works on Bifurcation theory, see $[\mathrm{A}]$ and the references therein). In this paper, after having given some generalities in Section 2, we discuss two examples. In Section 3 we consider a simple model of one dimensional random walk, and show that the stochastic attractor depends on the (non-unique) choice of the realization of a process as a random dynamical system. For a suitable realization we show that the attractor is a two-points set, corresponding to the equilibria of the system, which in this case are deterministic. In Section 4 we consider a more complex system, the Contact Process (see [Li1]). Its realization as a stochastic dynamical system is provided by the well known graphical construction. For a sufficiently large infection rate this system has two extremal invariant measures, one of which is concentrated in a deterministic equilibrium point. We show that, as for the random walk, the stochastic attractor is comprised of two equilibrium points, but in this case one of them is random. In particular this shows that, in a finite random time, one can obtain a sample, restricted to a finite space number of sites, from the upper invariant measure of the contact process, starting the system from a configuration with a finite number of infected sites, and updating only a finite number of sites (i.e., in a computer simulation, using a finite amount of memory). Here we note a further difference from the usual forward Markov Chain Monte Carlo: if the Contact Process is started from a configuration with a finite number of infected sites and evolves forward in time, with positive probability the system dies out without ever getting "close" to the upper invariant measure.

\section{Some preliminaries on Random Dynamical Sys- tems}

\subsection{General Definitions}

Definition 1. A Metric Dynamical System $\theta=\left((\Omega, \mathcal{F}, \mathbb{P}),\left(\theta_{t}\right)_{t \in \mathbb{T}}\right)$ with time $\mathbb{T}$ (where $\mathbb{T}=\mathbb{Z}$ or $\mathbb{R}$ ) is a probability space $(\Omega, \mathcal{F}, \mathbb{P})$ with a family of transformations $\left\{\theta_{t}: \Omega \rightarrow \Omega ; t \in \mathbb{T}\right\}$ such that:

1) $(t, \omega) \mapsto \theta_{t} \omega$ is measurable

2) $\left(\theta_{t}\right)_{t \in \mathbb{T}}$ is a one-parameter group, that is

$$
\theta_{0} \equiv \operatorname{id}_{\Omega}, \quad \theta_{t} \circ \theta_{s}=\theta_{t+s} \text { for all } s, t \in \mathbb{T}
$$

3) For every $t \in \mathbb{T}, \theta_{t}$ is measure-preservig, i.e.

$$
\mathbb{P}\left\{\theta_{t} \in B\right\}=\mathbb{P}(B) \text { for every } B \in \mathcal{F} .
$$

If we think of $\omega$ as a model for a random perturbation evolving in time, $\theta_{t} \omega$ is "the noise" acting on the system if the initial time is $t$. 
For the next definition we assume that $(X, d)$ is a Polish space (i.e. a separable complete metric space) and $\mathcal{B}(X)$ is its corresponding Borel $\sigma$-algebra.

Definition 2 . A Random Dynamical System with time $\mathbb{T}^{+}$and state space $X$, over a metric dynamical system $\theta$, is a measurable map

$$
\begin{aligned}
\varphi: \mathbb{T}^{+} \times \Omega \times X & \longrightarrow X, \\
(t, \omega, x) & \longmapsto \varphi(t, \omega, x) \equiv \varphi(t, \omega) x
\end{aligned}
$$

such that the family of maps $\{\varphi(t, \omega) ; t \in \mathbb{T}, \omega \in \Omega\}$ satisfies the cocycle property,

$$
\varphi(0, \omega)=\operatorname{id}_{X}, \quad \varphi(t+s, \omega)=\varphi\left(t, \theta_{s} \omega\right) \circ \varphi(s, \omega)
$$

for every $s, t \in \mathbb{T}$ and $\omega \in \Omega$.

We say that $\varphi$ is continuous if $\varphi(t, \omega): X \longrightarrow X$ is continuous for every fixed $t \in \mathbb{T}^{+}$ and $\omega \in \Omega$.

The state $\varphi\left(t, \theta_{s} \omega\right) x$ is the state of the system after a time $t$, started at time $s$ from the state $x$. The cocycle property

$$
\varphi(t+s, \omega) x=\varphi\left(t, \theta_{s} \omega\right) \varphi(s, \omega) x
$$

states that, if we start at time 0 from the state $x$, then the state of the system after a time $t+s$ is the same as if we start at time $s$ from the state $\varphi(s, \omega) x$ and wait a time $t$. This is just a semiflow property.

In references $[\mathrm{A}]$ and $[\mathrm{Ch}]$, several relations between random dynamical systems and Markov processes are discussed. Few essential facts are summarized in the following statements.

Definition 3 . Let $\mathbb{G}$ be the set of functions from $X$ to itself and $\mathcal{G}$ be the smaller $\sigma$-algebra on $\mathbb{G}$ for which the maps $\left\{\Phi_{x}: f \mapsto f(x) ; x \in X\right\}$ are measurable. A Random Dynamical system $\varphi$ has independent increments if, for all $n \in \mathbb{Z}^{+}$, and $\left\{t_{1}, \ldots, t_{n}\right\} \subset \mathbb{T}$, the random variables

$$
\varphi\left(t_{n}-t_{n-1}, \theta_{t_{n-1}} \cdot\right), \ldots, \varphi\left(t_{2}-t_{1}, \theta_{t_{1}} \cdot\right)
$$

(defined on $(\Omega, \mathcal{F})$ with values in $(\mathbb{G}, \mathcal{G}))$ are independent.

Proposition 1 . Suppose that $\varphi$ has independent increments. Then, for each fixed $x^{n}=\left(x_{1}, \ldots, x_{n}\right) \in X^{n}$, the n-point motion of $\varphi$, i.e., the stochastic process $\left(\varphi^{n}(t, \cdot) x^{n}\right)_{t \in \mathbb{T}^{+}}$, with $\varphi^{n}(t, \cdot) x^{n}=\left(\varphi(t, \cdot) x_{1}, \ldots, \varphi(t, \cdot) x_{n}\right)$ is an homogeneous Markov process with respect to its natural filtration and with transition probability $P_{t}\left(x^{n}, B\right)=\mathbb{P}\left(\varphi^{n}(t, \cdot) x^{n} \in\right.$ $B)$, where $t \in \mathbb{T}^{+}$and $B \in \mathcal{B}(X)^{\otimes n}$. Moreover, if $\varphi$ is continuous, then this process has the Feller property. 


\subsection{Random sets, equilibrium points and attractors}

In what follows we shall always suppose that $\varphi$ is a continuous RDS.

Definition 4 . Let $(X, d)$ be a Polish space and $(\Omega, \mathcal{F})$ a measurable space. A random set $\mathrm{D}$ is a set valued map

$$
D: \Omega \longrightarrow 2^{X}
$$

such that, for each $\omega \in \Omega, D(\omega)$ is a closed subset of $X$ and, for every $x \in X$ the map $\omega \mapsto d(x, D(\omega))$ is measurable.

A random compact set is a random set $D$ such that $D(\omega)$ is compact for each $\omega \in \Omega$.

Clearly, a deterministic set is a particular random set. Note that we can identify a random variable with values in $X$ with a random point, i.e. a random set $D$ such that $D(\omega)$ is a single point of $X$ for each $\omega \in \Omega$.

For general properties of random sets (or "measurable multifunctions") we refer to Castaing and Valadier [CV]. See also [HP]. We shall need the following assertions on whether a multifunction, i.e., a set valued map, is a random compact set. The proof follows from Theorem III.9 p.67 and from Lemma III.14, p.70 (for part a)) and Proposition III.4, p.63 (for part b)) of [CV].

Proposition 2 . Let $X$ be a Polish space. Then we have:

a) Let $f: \Omega \times X \longrightarrow X$ be a map such that $f(\omega, \cdot)$ is continuous for all $\omega \in \Omega$ and $f(\cdot, x)$ is measurable for all $x \in X$. Then, if $D$ is a random compact set in $X$, the map $f(\cdot, D): \omega \mapsto f(\omega, D(\omega))$ defines a random compact set in $X$.

b) If $\left\{D_{n} ; n \in \mathbb{Z}^{+}\right\}$is a family of random compact sets, then $\cap_{n} D_{n}$ is a random compact set in $X$.

Definition 5 . A random set (or, more generally, a multifunction) $D$ is said to be $\varphi$-invariant if, for every $t \in \mathbb{T}^{+}$and $\omega \in \Omega$, we have

$$
\varphi(t, \omega) D(\omega)=D\left(\theta_{t} \omega\right) .
$$

If in the above formula we have " $\subset$ " instead of "=" we say that $D$ is $\varphi$-forward invariant. We call equilibrium point a random point which is $\varphi$-invariant.

An equilibrium point $u$ is an analog of a fixed point for deterministic dynamical systems. In fact the stochastic process

$$
\{\varphi(t, \cdot) u(\cdot)\}_{t \geq 0}=\left\{u\left(\theta_{t} \cdot\right)\right\}_{t \geq 0}
$$

is a stationary process (because $\left(\theta_{t}\right)_{t \in \mathbb{T}}$ is measure preserving). Moreover, suppose that $\varphi$ is a random dynamical system which gives a realization of a Markov process (i.e. its one-point motions $\{\varphi(t, \cdot) x\}_{t \in \mathbb{T}^{+}}$, with $x \in X$, are homogeneous Markov processes). If $\varphi$ has an equilibrium point $u$ which measurable with respect to the "past" $\sigma$-field 
$\mathcal{F}^{-}=\sigma\left(\varphi\left(r, \theta_{-t}\right) ; 0 \leq r \leq t, t \in \mathbb{T}^{+}\right)$, then (2.1) is a Markov process, and so the distribution of the random variable $u$ is an invariant measure for the process.

This relation between $\mathcal{F}^{-}$-measurable equilibrium points and invariant measures can be generalized to more general $\varphi$-invariant random compact sets: for results in this direction see $[\mathrm{C}, \mathrm{CF}]$.

Next definitions introduce the notion of random attractors.

Definition 6 . For a fixed $\omega \in \Omega$, the function $t \mapsto \varphi\left(t, \theta_{-t} \omega\right) x$ is called the pull-back trajectory starting from $x$.

In the literature several definitions of random attractors can be found (see, for example, [Ch], [CS], [CF]). The definition below is an extension of the notion of global attractor given by H.Crauel and F. Flandoli in [CF].

Definition 7 . Let $\mathcal{B}$ be a family of subsets of $X$. A $\mathcal{B}$-random (pull-back) attractor for the $\operatorname{RDS} \varphi$ is a random compact set $A$ which is $\varphi$-invariant and attracts every $B \in \mathcal{B}$, i.e., for all $B \in \mathcal{B}$ we have

$$
\lim _{t \rightarrow+\infty} d\left(\varphi\left(t, \theta_{-t} \omega\right) B, D(\omega)\right)=0 \quad \mathbb{P}-\text { a.s. }
$$

where $d(A, B)=\sup _{x \in A} d(x, B)=\sup _{x \in A}\left\{\inf _{y \in B} d(x, y)\right\}$.

Note that in the definition of attraction we are considering the pull-back trajectories starting in $B$.

Following $[\mathrm{CF}]$, we say that $A$ is a global attractor for $\varphi$ if in the definition above $\mathcal{B}$ is the family of all bounded subsets of $X$.

In $[\mathrm{CF}]$ a general result about the existence of a global attractor for RDS is proved. In this paper we shall consider only RDS with a compact state space. In this case a stronger result holds and it has an elementary proof, which is an application of Proposition 3.6, p.369 of Crauel and Flandoli $[\mathrm{CF}]$ and that we sketch for the sake of completeness. We first need the following definition, in which the notation $\bar{B}$ denotes the closure of a subset $B$ of $X$ :

Definition 8 . Given a random set $D$, the (pull back) $\omega$-limit set of $D$ is the multifunction defined by

$$
\Omega_{D}(\omega)=\bigcap_{T \geq 0} \overline{\bigcup_{t \geq T} \varphi\left(t, \theta_{-t} \omega\right) D\left(\theta_{-t} \omega\right)}
$$

Remark 1 . The $\omega$-limit set of $D$ can be characterized as follows: $y \in \Omega_{D}(\omega)$ if and only if there exist sequences $\left\{t_{n}\right\}_{n} \subset \mathbb{T}^{+}$with $t_{n} \rightarrow+\infty$ and $\left\{x_{n}\right\}_{n} \subset X$ with $x_{n} \in D\left(\theta_{-t_{n}} \omega\right)$ for each $n$, such that $y=\lim _{n \rightarrow+\infty} \varphi\left(t_{n}, \theta_{-t_{n}} \omega\right) x_{n}$. 
Proposition 3 . Suppose that the RDS $\varphi$ has a compact state space $X$. Then $\Omega_{X}$, is a $\mathcal{B}$-attractor for every family of sets $\mathcal{B}$.

Proof. From Proposition 3.6, p.368 of $[\mathrm{CF}]$, follows that $\Omega_{X} \neq \emptyset$ and it is a $\varphi$ invariant multifunction which attracts every subset of $X$. Moreover, $\Omega_{X}$ is a random compact set. Indeed, since $\varphi$ is measurable and $\varphi(t, \omega)$ is continuous, from property a) of proposition 2 follows that, for each fixed $t$, the map $\omega \mapsto \varphi\left(t, \theta_{-t} \omega\right) X$ defines a random compact set. Moreover, the family of random sets $\left\{\varphi\left(t, \theta_{-t^{*}}\right) X ; t \geq 0\right\}$ is decreasing in $t$. Indeed, using the cocycle property, for each $h>0$ we have

$$
\begin{aligned}
\varphi\left(t+h, \theta_{-t-h} \omega\right) X & =\varphi\left(t, \theta_{-t} \omega\right) \varphi\left(h, \theta_{-t-h} \omega\right) X \\
& \subset \varphi\left(t, \theta_{-t} \omega\right) X .
\end{aligned}
$$

Then,

$$
\Omega_{X}(\omega)=\bigcap_{t>0} \varphi\left(t, \theta_{-t} \omega\right) X=\bigcap_{n \in \mathbb{Z}^{+}} \varphi\left(n, \theta_{-n} \omega\right) X
$$

and hence, by property b) of proposition $2, \Omega_{X}$ is a random compact set.

Note that $\Omega_{X}$ is the minimal (with respect to set inclusion) random compact set which attracts $X$. Indeed, if $A$ is a random compact set and attracts $X$, we have, for all $\omega, d\left(\Omega_{X}(\omega), A(\omega)\right)=d\left(\cap_{n} \varphi\left(n, \theta_{-n} \omega\right) X, A(\omega)\right) \leq d\left(\varphi\left(n, \theta_{-n} \omega\right) X, A(\omega)\right)$ for all $n$ and hence $\Omega_{X}(\omega) \subset A(\omega)$. However, as we shall see in the examples, $\Omega_{X}$ need not be the minimal attractor for each $\mathcal{B}$.

\subsection{Monotone Random Dynamical Systems}

An extensive treatment of monotone RDS having a partially ordered separable Banach space as state space can be found in Chueshov [Ch]. Here we assume that the state space $X$ is a partially ordered Polish space and we rewrite some results by Arnold and Chueshov $[\mathrm{AC}]$ which follow from this assumption.

Definition 9 . A Random Dynamical System $\varphi$ is said to be monotone if

$$
x \leq y \text { implies } \varphi(t, \omega) x \leq \varphi(t, \omega) y \text { for all } t \in \mathbb{T}^{+} \text {and } \omega \in \Omega
$$

For a monotone RDS, if the state space is such that there exist two elements $\overline{0}, \overline{1} \in X$ with $\overline{0} \leq x \leq \overline{1}$ for all $x \in X$ and every monotone sequence in $X$ converges to a point of $X$, then there is a simple way to obtain two equilibrium points. In fact, if we fix $\omega \in \Omega$, then the family of points in $X\left\{\varphi\left(t, \theta_{-t} \omega\right) \overline{1}\right\}_{t \in \mathbb{T}^{+}}$is decreasing in $t$ :

$$
s \leq t \Rightarrow \varphi\left(s, \theta_{-s} \omega\right) \overline{1} \geq \varphi\left(t, \theta_{-t} \omega\right) \overline{1} .
$$

This is easily seen by applying the cocycle property and the monotonicity of $\varphi$ :

$$
\begin{aligned}
\varphi\left(t, \theta_{-t} \omega\right) \overline{1} & =\varphi\left(s, \theta_{-s} \omega\right) \varphi\left(t-s, \theta_{-t} \omega\right) \overline{1} \\
& \leq \varphi\left(s, \theta_{-s} \omega\right) \overline{1} .
\end{aligned}
$$


In the same way we see that $\left\{\varphi\left(t, \theta_{-t} \omega\right) \overline{0}\right\}_{t \in \mathbb{T}^{+}}$is increasing in $t$.

As a consequence, for every $\omega \in \Omega$ the following limits exist

$$
\sigma^{+}(\omega):=\lim _{t \rightarrow+\infty} \varphi\left(t, \theta_{-t} \omega\right) \overline{1} \quad \text { and } \quad \sigma^{-}(\omega):=\lim _{t \rightarrow+\infty} \varphi\left(t, \theta_{-t} \omega\right) \overline{0}
$$

The following Proposition can be found in $[\mathrm{AC}]$, Theorem1, p.272. We include here its simple and nice proof.

Proposition 4 . The random variables $\sigma^{+}$e $\sigma^{-}$are equilibrium points for $\varphi$, i.e. $\varphi(t, \omega) \sigma^{+}(\omega)=\sigma^{+}\left(\theta_{t} \omega\right)$ and $\varphi(t, \omega) \sigma^{-}(\omega)=\sigma^{-}\left(\theta_{t} \omega\right)$ for all $t \in \mathbb{T}^{+}$and $\omega \in \Omega$. Moreover, they are measurable with respect to the past sigma-algebra $\mathcal{F}^{-}$.

Proof. If we fix $t$ and $\omega$, by the continuity of $\varphi(t, \omega)$ follows

$$
\begin{aligned}
\varphi(t, \omega) \sigma^{+}(\omega) & =\varphi(t, \omega)\left(\lim _{s \rightarrow+\infty} \varphi\left(s, \theta_{-s} \omega\right) \overline{1}\right) \\
& =\lim _{s \rightarrow+\infty} \varphi(t, \omega) \varphi\left(s, \theta_{-s} \omega\right) \overline{1}
\end{aligned}
$$

and by the cocycle property,

$$
\begin{aligned}
& =\lim _{s \rightarrow+\infty} \varphi\left(t+s, \theta_{-s} \omega\right) \overline{1} \\
& =\lim _{s \rightarrow+\infty} \varphi\left(t+s, \theta_{-(t+s)} \theta_{t} \omega\right) \overline{1}=\sigma^{+}\left(\theta_{t} \omega\right) .
\end{aligned}
$$

Measurability with respect to $\mathcal{F}^{-}$follows immediately from the definition of $\sigma^{-}$and $\sigma^{+}$

Note that from this proposition it follows that, if $\varphi$ gives a realization of a Markov process, the distributions of the random variables $\sigma^{-}$and $\sigma^{+}$are invariant measures for the process.

\section{Random attractors for two RDS's realizing a one dimensional random walk}

As a first example, we illustrate how two different realizations of a Markov process as random dynamical system give different attractors.

Our state space will be $X=\mathbb{Z} \cup\{-\infty,+\infty\}$ with the compactifying topology, which is induced by the metric $d(x, y)=|g(x)-g(y)|$, where $g$ is the function defined on $X$ as follows: $g(z)=\frac{z}{1+|z|}$ if $z \in \mathbb{Z}$ and $g( \pm \infty)= \pm 1$.

Let us consider a symmetric (continuous time) random walk on $X$, i.e., a Markov process on $X$ with infinitesimal generator $L=\left(L_{x y}\right)_{x, y \in X}$ such that, for $x, y \in X$ with $x \neq y$

$$
L_{x y}= \begin{cases}\frac{1}{2} & \text { if } x, y \in \mathbb{Z} \text { and }|x-y|=1 \\ 0 & \text { otherwise }\end{cases}
$$


This process has two invariant measures, the Dirac measures concentrated at $-\infty$ and $+\infty$.

A way of realizing the process as the one-point motion of a random dynamical system is by taking as probability space the space of all the realizations of two independent Poisson processes, $N^{+}$and $N^{-}$, of intensity $\frac{1}{2}$. Then the one-point motion of the RDS will be defined as follows: at each jump time of the process $N^{+}\left(\operatorname{resp} . N^{-}\right)$, the current state $y$ will jump at $y+1$ (resp. $y-1$ ). In other words, we can construct the one-point motion starting at 0 and put, for each $x \in \mathbb{Z}, \varphi(t, \omega) x=x+\varphi(t, \omega) 0$.

More precisely, in what follows we let $\mathcal{S}$ be the set of locally finite subsets of $\mathbb{R}$, i.e., those sets $E \subset \mathbb{R}$ such that, for all $a, b \in \mathbb{R}, E \cap[a, b]$ has a finite number of points. Each element $E$ of $\mathcal{S}$ can be identified with the càdlag step function

$$
f_{E}(t)= \begin{cases}|E \cap(0, t]| & \text { if } t \geq 0 \\ -|E \cap(t, 0]| & \text { if } t<0\end{cases}
$$

where, the notation $|E|$ stands for the cardinality of $E$.

With this identification, $\mathcal{S}$ may be provided with the metric induced by the Skorohod metric on càdlág functions from $\mathbb{R}$ to $\mathbb{R}$, and with the corresponding Borel $\sigma$-field $\mathcal{B}(\mathcal{S})$ (see $[\mathrm{EK}]$ as a reference on the Skorohod metric). For $t \in \mathbb{R}$, the map $\vartheta_{t}: \mathcal{S} \rightarrow \mathcal{S}$ is the natural shift $\vartheta_{t}(E)=E-t$. Note that the map $(t, E) \mapsto \vartheta_{t}(E)$ is jointly continuous. By a Poisson process with intensity $\lambda>0$ we mean a $\mathcal{S}$-valued random variable $N$ such that for any $A, B$ disjoint Borel subsets of $\mathbb{R}$, the real-valued random variables $|N \cap A|$ and $|N \cap B|$ are independent Poisson random variables with parameters $\lambda m(A)$ and $\lambda m(B)$ respectively, where $m(\cdot)$ is the Lebesgue measure. It follows from this definition that, for all $t \in \mathbb{R}, N$ and $\vartheta_{t} N$ have the same distribution.

Therefore we take as probability space $\Omega=\mathcal{S} \times \mathcal{S}$, with the product $\sigma$-field $\mathcal{B}(\mathcal{S}) \otimes$ $\mathcal{B}(\mathcal{S})$, which we denote by $\mathcal{F}$, and $\mathbb{P}$ being the product measure $\mathbb{P}=P^{\frac{1}{2}} \otimes P^{\frac{1}{2}}$, where $P^{\frac{1}{2}}$ denotes the law of a Poisson process of intensity $\frac{1}{2}$. If, for $\omega \in \Omega$ with $\omega=\left(\omega^{+}, \omega^{-}\right)$, we define $\theta_{t} \omega=\left(\vartheta_{t} \omega^{+}, \vartheta_{t} \omega^{-}\right)$, then $\theta_{t}$ is a measure preserving transformation, so that $\left(\Omega, \mathcal{F}, \mathbb{P},\left(\theta_{t}\right)_{t \in \mathbb{R}}\right)$ is a metric dynamical system.

Now, following $[\mathrm{Br}]$, for each $x \in \mathbb{Z}$, let we define the sequence $\left\{\left(\tau_{n}(\omega), X_{n}(\omega, x)\right)\right\}_{n \geq 0}$ defined as follows:

i) $\left(\tau_{0}(\omega), X_{0}(\omega, x)\right)=(0, x)$

ii) Given $\left(\tau_{n}(\omega), X_{n}(\omega, x)\right)$, we set

$$
\begin{gathered}
\tau_{n+1}(\omega)=\inf \left\{t:\left(\omega^{+} \cup \omega^{-}\right) \cap\left(\tau_{n}(\omega), t\right] \neq \emptyset\right\} \\
(\text { with the convention } \inf \emptyset=\infty), \\
X_{n+1}(\omega, x)=\left\{\begin{array}{l}
X_{n}(\omega, x)+1 \text { for } \tau_{n+1}(\omega) \in \omega^{+} \\
X_{n}(\omega, x)-1 \text { for } \tau_{n+1}(\omega) \in \omega^{-}
\end{array}\right.
\end{gathered}
$$


and $X_{n+1}(\omega, x)=X_{n}(\omega, x)$ for $\omega$ in the $\mathbb{P}$-null set $\left\{\omega: \tau_{n+1}(\omega) \in \omega^{+} \cap \omega^{-}\right\} \cup$ $\left\{\omega: \tau_{n+1}(\omega)=\infty\right\}$

Now, we set

$$
\varphi(t, \omega) x=\sum_{n \geq 0} X_{n}(\omega, x) \mathbf{1}_{\left[\tau_{n}(\omega), \tau_{n+1}(\omega)\right)}(t)
$$

and, for $x= \pm \infty, \varphi(t, \omega) x=x$ for all $t$ and $\omega$.

Note that for the sequence defined above we have, for all $x \in \mathbb{Z}$ and $n \geq 0, X_{n}(\omega, x)=$ $x+X(\omega, 0)$, and so $\varphi(t, \omega) x=x+\varphi(t, \omega) 0$. For each $x \in X$, the stochastic process $\{\varphi(t, \cdot) x\}_{t \in \mathbb{R}^{+}}$is a Markov process starting from state $x$ and having $L$ as its infinitesimal generator (for the proof see, for example, $[\mathrm{Br}]$ ). Moreover, the map $\varphi: \mathbb{R}^{+} \times \Omega \times X \longrightarrow X$ defines a monotone random dynamical system over the metric dynamical system $\left(\Omega, \mathcal{F}, \mathbb{P},\left(\theta_{t}\right)_{t \in \mathbb{R}}\right)$. Indeed, for each $x,\{\varphi(t, \cdot) x\}_{t \in \mathbb{R}^{+}}$is a cádlág stochastic process and hence $(t, \omega, x) \longmapsto \varphi(t, \omega) x$ is measurable. For the cocycle property, let $k \geq 0$ be such that $s \in\left[\tau_{k}(\omega), \tau_{k+1}(\omega)\right)$, so that $\varphi(s, \omega) x=X_{k}(\omega, x)$. By the construction of the RDS it follows readily that $X_{n}\left(\theta_{s} \omega, X_{k}(\omega, x)\right)=X_{n+k}(\omega, x)$ and $\tau_{n}\left(\theta_{s} \omega\right)+s=\tau_{n+k}(\omega)$. Therefore

$$
\begin{aligned}
\varphi\left(t, \theta_{s} \omega\right) \varphi(s, \omega) x=\sum_{n \geq 0} X_{n}\left(\theta_{s} \omega, X_{k}(\omega, x)\right) I_{\left[\tau_{n}\left(\theta_{s} \omega\right), \tau_{n+1}\left(\theta_{s} \omega\right)\right)}(t) \\
\quad=\sum_{n \geq 0} X_{n+k}(\omega, x) I_{\left[\tau_{n+k}(\omega), \tau_{n+k+1}(\omega)\right)}(t+s)=\varphi(t+s, \omega) x .
\end{aligned}
$$

Monotonicity of $\varphi$ is obvious. Since the state space $X$ is compact, from Proposition 3 it follows that $\Omega_{X}$ is a $\mathcal{B}$-random attractor for $\varphi$, for every family of sets $\mathcal{B}$. In particular, (see remark 1) $\Omega_{X}$ contains all the limit points of the pull-back trajectories of the system.

Now, let us fix $x \in \mathbb{Z}$ and consider the pull-back process $\left\{\varphi\left(t, \theta_{-t^{\cdot}}\right) x\right\}_{t \in \mathbb{R}^{+}}$. If we denote by $N^{+}$and $N^{+}$the canonical projections on $\Omega$, for $s, t \in \mathbb{R}$ let $\mathcal{F}_{s, t}$ be the $\sigma$-field generated by the random variables $\left\{N^{+} \cap(u, v], N^{-} \cap(u, v] ; s \leq u \leq v \leq t\right\}$. Then $\left\{\varphi\left(t, \theta_{-t^{*}}\right) x\right\}_{t \in \mathbb{R}^{+}}$is $\left(\mathcal{F}_{-t, 0}\right)_{t \in \mathbb{R}^{+-}}$adapted and it is a random walk with the same generator as $\{\varphi(t, \cdot) x\}_{t \in \mathbb{R}^{+}}$.

Indeed, for each $x, y \in \mathbb{Z}$ we have $\varphi(t, \omega) x=\varphi(t, \omega) y+(x-y)$, therefore, for each $t, h \geq 0, y, z \in \mathbb{Z}$ and $A \in \mathcal{F}_{-t, 0}$

$$
\begin{aligned}
& \mathbb{P}\left\{\varphi\left(t+h, \theta_{-t-h} \cdot\right) x=y \mid \varphi\left(t, \theta_{-t} \cdot\right) x=z, A\right\}= \\
& \mathbb{P}\left\{\varphi\left(t, \theta_{-t^{\cdot}}\right) \varphi\left(h, \theta_{-t-h} \cdot\right) x=y \mid \varphi\left(t, \theta_{-t^{\cdot}}\right) x=z, A\right\}= \\
& \mathbb{P}\left\{\varphi\left(t, \theta_{-t} \cdot\right) x+\varphi\left(h, \theta_{-t-h} \cdot\right) x-x=y \mid \varphi\left(t, \theta_{-t} \cdot\right) x=z, A\right\}= \\
& \mathbb{P}\left\{\varphi\left(h, \theta_{-t-h^{\cdot}}\right) x=x+y-z \mid \varphi\left(t, \theta_{-t} \cdot\right) x=z, A\right\}= \\
& \mathbb{P}\left\{\varphi\left(h, \theta_{-t-h} \cdot\right) x=x+y-z\right\}=\mathbb{P}\{\varphi(h, \cdot) x=x+y-z\} .
\end{aligned}
$$

where the last two equalities follow from the fact that $\varphi\left(h, \theta_{-t-h} \cdot\right) x$ is $\mathcal{F}_{-t-h,-t^{-}}$ measurable (and $\mathcal{F}_{-t-h,-t}$ is independent of $\mathcal{F}_{-t, 0}$ ) and the measure preserving property of $\theta_{-t-h}$. 
Then $\left\{\varphi\left(t, \theta_{-t}\right) x\right\}$ is a symmetric random walk and, since a symmetric random walk visits almost surely infinitely many times each point of $\mathbb{Z}$, it follows that, for $\mathbb{P}$-almost all $\omega$, each point of $X$ is a limit point for the pull-back trajectory $t \mapsto \varphi\left(t, \theta_{-t} \omega\right) x$ and hence, since these limit points are contained in $\Omega_{X}, \Omega_{X}$ is given ( $\mathbb{P}$-almost surely) by the whole state space. Note that, since $-\infty$ and $+\infty$ are fixed points for $\varphi$, this is a first trivial example in which $\Omega_{X}$ is not the minimal attractor for every collection of subsets of $X$. A less trivial example will be shown in next section.

Now we consider another RDS $\phi$ which gives a realization of the same random walk. In this case the noise depends also on the position in space, i.e., it will be described by a countable family of independent Poisson processes $\left(N^{x,+}, N^{x,-} ; x \in \mathbb{Z}\right)$. The one-point motion of $\phi$ will be such that, at each jump time of $N^{x,+}$ (respectively $N^{x,-}$ ), if the current position is $x$, it will jump at $x+1$ (respectively $x-1$ ).

The construction of the RDS is similar to the one given above. Here the metric dynamical system is given by $\Omega=\mathcal{S}^{\mathbb{Z}} \times \mathcal{S}^{\mathbb{Z}}, \mathcal{F}=(\mathcal{B}(\mathcal{S}) \otimes \mathcal{B}(\mathcal{S}))^{\otimes \mathbb{Z}}, \mathbb{P}=\left(P^{\frac{1}{2}} \otimes P^{\frac{1}{2}}\right)^{\otimes \mathbb{Z}}$ and, for $\omega=\left(\omega^{x,+}, \omega^{x,-} ; x \in \mathbb{Z}\right) \in \Omega, \theta_{t}(\omega)=\left(\vartheta_{t} \omega^{x,+}, \vartheta_{t} \omega^{x,-} ; x \in \mathbb{Z}\right)$.

We consider the sequence $\left\{\left(\tau_{n}(\omega), X_{n}(\omega, x)\right)\right\}_{n}$ defined by:

$$
\begin{aligned}
& \left(\tau_{0}(\omega), X_{0}(\omega, x)\right)=(0, x) \\
& \tau_{n+1}(\omega)=\inf \left\{t:\left(\omega^{X_{n}(\omega, x),+} \cup \omega^{X_{n}(\omega, x),-}\right) \cap\left(\tau_{n}(\omega), t\right] \neq \emptyset\right\} \\
& X_{n+1}(\omega, x)= \begin{cases}X_{n}(\omega, x)+1 & \text { if } \tau_{n+1}(\omega) \in \omega^{X_{n}(\omega, x),+} \\
X_{n}(\omega, x)-1 & \text { if } \tau_{n+1}(\omega) \in \omega^{X_{n}(\omega, x),-}\end{cases}
\end{aligned}
$$

Then the map $\phi$ will be defined as in (3.1). The same arguments used for $\varphi$ show that $\phi$ is a monotone RDS and its one-point motions are random walks with infinitesimal generator given by $L$.

With this realization, however, the behavior of the pull-back trajectories is different. We establish the following result.

Proposition 5 . Let $\phi$ be defined as above. Then, for each $M \in \mathbb{Z}^{+}$there is a random time $t_{M}$ such that, for $\mathbb{P}$-almost all $\omega, t_{M}(\omega)$ is finite and

$$
t \geq t_{M}(\omega) \Rightarrow \inf _{x \in \mathbb{Z}}\left|\phi\left(t, \theta_{-t} \omega\right) x\right| \geq M
$$

Proof. For each $t \geq 0$, let us define the random variables

$$
\begin{aligned}
& Z_{t}^{1}=\inf \left\{x: \phi\left(t, \theta_{-t^{*}}\right) x \geq M\right\} \\
& Z_{t}^{2}=\sup \left\{x: \phi\left(t, \theta_{-t^{*}}\right) x \leq-M\right\}
\end{aligned}
$$

Note that $Z_{t}^{1} \geq Z_{t}^{2}+1$ for each $t \geq 0$ and, $Z_{t}^{1}(\omega)=Z_{t}^{2}(\omega)+1$ if and only if $\left|\phi\left(t, \theta_{-t} \omega\right) x\right| \geq M$ for each $x \in \mathbb{Z}$ : indeed, if $Z_{t}^{1}(\omega)=Z_{t}^{2}(\omega)+1$, then for each $x \in \mathbb{Z}$ we have $x \geq Z_{t}^{1}(\omega)$ or $x \leq Z_{t}^{2}(\omega)$. Then, from the monotonicity of $\phi$ follows 
that, for each $x \in \mathbb{Z}, \phi\left(t, \theta_{-t} \omega\right) x \geq M$ or $\phi\left(t, \theta_{-t} \omega\right) x \leq-M$; on the other hand, if this last property holds it is clear that $Z_{t}^{1}(\omega)=Z_{t}^{2}(\omega)+1$.

On the other hand, if there exists some $\bar{s} \in \mathbb{R}^{+}$such that $Z_{\bar{s}}^{1}(\omega)=Z_{\bar{s}}^{2}(\omega)+1$, then we have, $Z_{t}^{1}(\omega)=Z_{t}^{2}(\omega)+1$ for all $t \geq \bar{s}$ or, equivalently, $\left|\phi\left(t, \theta_{-t} \omega\right) x\right| \geq M$ for all $x \in \mathbb{Z}$ and $t \geq \bar{s}$. This follows easily from the cocycle property: indeed, if $t>\bar{s}$, then for each $x \in \mathbb{Z}$ we have $\left|\phi\left(t, \theta_{-t} \omega\right) x\right|=\left|\phi\left(\bar{s}, \theta_{-\bar{s}}\right) \phi\left(t-\bar{s}, \theta_{-t} \omega\right) x\right| \geq M$. Then, it is sufficient to prove that, for each $\omega$, there exists such $\bar{s}$; in order to do that, we shall prove that the stochastic process $\left\{\left(Z_{t}^{1}, Z_{t}^{2}\right)\right\}_{t>0}$, which has the set $C=\left\{(x, y) \in \mathbb{Z}^{2}: x=y+1\right\}$ as an absorbing barrier, is a symmetric random walk on $\mathbb{Z}^{2}$ starting at $(M,-M)$.

The process $\left\{\left(Z_{t}^{1}, Z_{t}^{2}\right)\right\}_{t \geq 0}$ is $\left(\mathcal{F}_{-t, 0}\right)$-adapted, where, for $s, t \in \mathbb{R}, \mathcal{F}_{s, t}$ is the $\sigma$-field generated by the family of random variables $\left\{N^{x,+} \cap(u, v], N^{x,-} \cap(u, v] ; s \leq u \leq v \leq\right.$ $t, x \in \mathbb{Z}\}$. Moreover, the monotonicity of $\phi$ implies that, for each $y \in \mathbb{Z}$

$$
Z_{t}^{1}(\omega)=y \Leftrightarrow \phi\left(t, \theta_{-t} \omega\right) y \geq M \text { and } \phi\left(t, \theta_{-t} \omega\right)(y-1)<M .
$$

Consider now $t, h \geq 0, A \in \mathcal{F}_{-t, 0}$, and $y, z, u, v \in \mathbb{Z}$ such that $y \geq u+1, z \geq v+1$.

$$
\begin{aligned}
& \mathbb{P}\left\{Z_{t+h}^{1}=y, Z_{t+h}^{2}=u \mid Z_{t}^{1}=z, Z_{t}^{2}=v, A\right\}= \\
& \mathbb{P}\left\{\phi\left(t+h, \theta_{-t-h} \cdot\right) y \geq M, \phi\left(t+h, \theta_{-t-h} \cdot\right)(y-1)<M,\right. \\
& \left.\quad \phi\left(t+h, \theta_{-t-h} \cdot\right) u-M, \phi\left(t+h, \theta_{-t-h} \cdot\right)(u+1)>-M \mid Z_{t}^{1}=z, Z_{t}^{2}=v, A\right\}= \\
& \mathbb{P}\left\{\phi\left(t, \theta_{-t} \cdot\right) \phi\left(h, \theta_{-t-h} \cdot\right) y \geq M, \phi\left(t, \theta_{-t} \cdot\right) \phi\left(h, \theta_{-t-h} \cdot\right)(y-1)<M,\right. \\
& \left.\quad \phi\left(t, \theta_{-t} \cdot\right) \phi\left(h, \theta_{-t-h} \cdot\right) u \leq M, \phi\left(t, \theta_{-t} \cdot\right) \phi\left(h, \theta_{-t-h} \cdot\right)(u+1)>-M \mid Z_{t}^{1}=z, Z_{t}^{2}=v\right\}= \\
& \mathbb{P}\left\{\phi\left(h, \theta_{-t-h} \cdot\right) y \geq Z_{t}^{1}, \phi\left(h, \theta_{-t-h} \cdot\right)(y-1)<Z_{t}^{1},\right. \\
& \left.\quad \phi\left(h, \theta_{-t-h} \cdot\right) u \leq Z_{t}^{2}, \phi\left(h, \theta_{-t-h} \cdot\right)(u+1)>Z_{t}^{2} \mid Z_{t}^{1}=z, Z_{t}^{2}=v\right\}= \\
& \quad \mathbb{P}\{\phi(h, \cdot) y \geq z, \phi(h, \cdot)(y-1)<z, \phi(h, \cdot) u \leq v, \phi(h, \cdot)(u+1)>v\},
\end{aligned}
$$

where we have used monotonicity, the independence between $\phi\left(h, \theta_{-t-h} \cdot\right) y$ and $\mathcal{F}_{-t, 0}$, and the measure preserving property of $\theta_{-t-h}$. This shows that $\left(Z_{t}^{1}, Z_{t}^{2}\right)$ is a Markov process. To determine its infinitesimal generator we compute the asymptotics of the probability in (3.3) as $h \downarrow 0$. We begin by claiming that the probability in (3.3) is $o(h)$ for $|y-z|+|u-v| \geq 2$. Suppose first $|y-z| \geq 2$. If $z \geq y+2$ then $(3.3) \leq \mathbb{P}\{\phi(h, \cdot) y \geq z\}=o(h)$, since the event $\{\phi(h, \cdot) y \geq z\}$ requires at least two jumps of the random walk $\{\phi(t, \cdot) y\}_{t \geq 0}$ in a time interval of length $h$. Similarly, if $z \leq y-2$, then $(3.3) \leq \mathbb{P}\{\phi(h, \cdot)(y-1)<z\}=o(h)$, and the same argument applies for $|u-v| \geq 2$.

We are just left to the case $|y-z|=1,|u-v|=1$.

Suppose first that $z=v+1$, i.e. $(z, v) \in C$. Then the probability in (3.3) is different from zero if and only if $y=u+1$ and it is given by $\mathbb{P}\{\phi(h, \cdot) y \geq z, \phi(h, \cdot)(y-1)<z\}$.

In the case $z=y+1$ we have

$$
\begin{aligned}
& \mathbb{P}\{\phi(h, \cdot) y \geq y+1, \phi(h, \cdot)(y-1)<y+1\}= \\
& \mathbb{P}\{\phi(h, \cdot) y \geq y+1, \phi(h, \cdot)(y-1)=y-1\}+o(h)=\frac{1}{2} h+2 o(h),
\end{aligned}
$$


and the same result can be obtained if $z=y-1$.

Now, suppose that $z>v+1$. To fix ideas, let $z=y+1, v=u+1$; all other cases can be dealt with similarly. The quantity in (3.3) is smaller or equal than

$\mathbb{P}\{\phi(h, \cdot) y \geq y+1, \phi(h, \cdot)(u+1)>u+1\} \mathbb{P}\{\phi(h, \cdot) y=y+1, \phi(h, \cdot)(u+1)=u+2\}+o(h)$.

Note that, since we have assumed $z>v+1$, we have $y>u+1$. Thus the event $\{\phi(h, \cdot) y=y+1, \phi(h, \cdot)(u+1)=u+2\}$ involves at least one jump for each of two independent Poisson processes $N^{y,+}, N^{u+1,+}$ in the time interval $(0, h]$, from which it follows that its probability is of order $h^{2}$.

We now compute the asymptotics of (3.3) for $|y-z|+|u-v|=1$. Let us take, for instance, $y=z-1, u=v$. We write the event in (3.3) as $\{\phi(h, \cdot) y \geq y+1, A\}$, where $A=\{\phi(h, \cdot)(y-1)<y+1, \phi(h, \cdot) u \leq u, \phi(h, \cdot)(u+1)>u\}$. Note that the events $\{\phi(h, \cdot) y \geq y+1\}$ and $A^{C}=\Omega \backslash A$ require respectively at least one jump time of the process $N^{y,+}$ and at least one jump time of one of the processes $N^{y-1,+}, N^{u,+}, N^{u+1,-}$ in the time interval $(0, h]$, so their intersection (since the Poisson processes above are independent) has probability of order $h^{2}$. Then

$$
\begin{aligned}
(3.3)= & \mathbb{P}\{\phi(h, \cdot) y \geq y+1\}-\mathbb{P}\left\{\phi(h, \cdot) y \geq y+1, A^{C}\right\}= \\
& \mathbb{P}\{\phi(h, \cdot) y \geq y+1\}+o(h)= \\
& \mathbb{P}\{\phi(h, \cdot) y=y+1\}+2 o(h)=\frac{1}{2} h+2 o(h) .
\end{aligned}
$$

All the other cases can be treated similarly.

Thus we have shown that $\left(Z_{t}^{1}, Z_{t}^{2}\right)$ is a Markov process whose infinitesimal generator is that of two coalescing random walks.

Now, to complete the proof, we observe that, by well known properties of random walks, there exists a random time $t_{M}$ such that, for $\mathbb{P}$-almost all $\omega, t_{M}(\omega)<\infty$ and $\left(Z_{t}^{1}(\omega), Z_{t}^{2}(\omega)\right) \in C$ for all $t \geq t_{M}(\omega)$.

As a consequence of the proposition above, we have

Corollary 1 . The deterministic set $\{-\infty,+\infty\}$ is a $\mathcal{B}$ - attractor for $\phi$, for every family $\mathcal{B}$ of subsets of $X$.

\section{Random attractor for a RDS realizing the Con- tact Process}

A Spin System is a Markov process with values in $X=\{0,1\}^{\mathbb{Z}^{d}}$. Many spin systems have been studied by representing them as RDS (this representation is known as graphical construction, see [Li1] and [Li2] as a reference); therefore we are interested in describing random attractors for these systems. Note that, given a RDS which realizes 
a spin system, since $X$ is compact, Proposition 3 holds, and therefore, for any family $\mathcal{B}$ of subsets of the state space, $\Omega_{X}$ is a $\mathcal{B}$-attractor for the RDS.

The aim of this section is to study the "pull-back" behavior of the supercritical Contact process (which is a particular monotone Spin System). We will first give a representation of the Contact Process as a monótone RDS and then we will study the limiting behavior of expressions like $\varphi\left(t, \theta_{-t} \omega\right) \xi$. We will see that this system has two equilibrium points which "attract" all the local subsets of the state space (see below for the definition of local set).

Now we give a short summary of some facts concerning the Contact Process. This process is a continuous time Markov process which can be thought of as a model for the spread of an infection. A collection of individuals is represented by the vertices of the integer lattice $\mathbb{Z}^{d}$. Each of these individuals can be infected or healthy. The state space of the process is $X=\{0,1\}^{\mathbb{Z}^{d}}$, where, if $\xi \in X$, by $\xi(x)=1$ we mean that the site $x$ is infected and by $\xi(x)=0$ we mean that the site $x$ is healthy. We provide $X$ with the product of the discrete topology on $\{0,1\}$, which is induced by the metric $d(\eta, \xi)=\sum_{x \in \mathbb{Z}^{d}}|\eta(x)-\xi(x)| 2^{-|x|}$, and it makes $X$ a Polish space. A partial order is given by $\eta \leq \xi \Longleftrightarrow \eta(x) \leq \xi(x)$ for all $x \in \mathbb{Z}^{d}$; the minimal and maximal states, with respect to this order, are given respectively by the two constant configurations $\overline{0}$ and $\overline{1}$ (the "all 0" and "all 1" configurations). The time evolution of this process may be described through its infinitesimal generator $A$. It is an (unbounded) operator defined on the set of functions $\mathcal{D}(X):=\left\{f \in \mathcal{C}(X): \sum_{x \in \mathbb{Z}^{d}} \sup _{\eta \in X}\left|f\left(\xi^{x}\right)-f(\xi)\right|<\infty\right\}$ by

$$
A f(\eta)=\sum_{x \in \mathbb{Z}^{d}} c(x, \xi)\left[f\left(\xi^{x}\right)-f(\xi)\right]
$$

where $\xi^{x} \in X$ is the configuration given by $\xi^{x}(y)=\xi(y)$ for each $y \neq x$, and $\xi^{x}(x)=$ $1-\xi(x)$, and

$$
c(x, \xi)= \begin{cases}1 & \text { if } \xi(x)=1 \\ \lambda \sum_{y:|y-x|=1} \xi(y) & \text { if } \xi(x)=0\end{cases}
$$

The quantity $c(x, \xi)$ is the "rate" at which a transition from $\xi$ to $\xi^{x}$ occurs. Its intuitive meaning is that, if in the site $x$ there is an infected individual, it will recover after a random time, exponentially distributed with parameter 1 , while if this individual is healthy, it will be infected after a random time with exponential distribution, whose parameter is proportional to the number of infected nearest neighbors of $x$.

Given the linear operator $A$ in (4.1), its closure is a Markov generator and the set $\mathcal{D}(X)$ is a core for $A$ (for the proof see [Li1]).

From now on, we will consider the Contact process in dimension 1.

The main result concerning convergence to equilibrium of the contact process is the following (see [Li1, Li2])

Theorem 1 . Let $\left(S_{t}\right)_{t \in \mathbb{R}^{+}}$be the semigroup generated by the operator in (4.1), and denote by $\nu S_{t}$ the action of the semigroup on probability measures on $X$. There exist a critical value $\lambda_{c}>0$ such that 
i) if $\lambda \leq \lambda_{c}$ then $\nu S_{t} \longrightarrow \delta_{\overline{0}}$ weakly, for every probability $\nu$. In particular, $\delta_{\overline{0}}$ is the unique stationary measure.

ii) (Complete convergence Theorem) If $\lambda>\lambda_{c}$, then there exist a constant $\alpha$, depending on $\nu$, such that $\nu S_{t} \longrightarrow \alpha \delta_{\overline{0}}+(1-\alpha) \mu^{+}$weakly, where $\mu^{+}$is the "upper invariant measure", i.e. the measure obtained as weak limit of $\delta_{\overline{1}} S_{t}$, as $t \rightarrow+\infty$. In particular, $\left\{\delta_{\overline{0}}, \mu^{+}\right\}$is the set of extremal stationary measures. Moreover $\alpha=0$ for $\nu=\delta_{\xi}$, where $\xi \in X$ is such that $\sum_{x} \xi(x)=+\infty$.

\subsection{The contact process as a random dynamical system: the graphical construction}

We are now ready to realize the contact process as a random dynamical system. This is done through the so-called graphical construction. The general argument is well known. However, some issues - such as measurability, continuity ...- are usually not addressed in the literature. For this reason we give here some details of the construction.

For each site $x \in \mathbb{Z}$ we consider three independent Poisson Processes: $N^{x}$, with intensity 1, whose points represent the "recovery times" (i.e. times in which, if the site $\mathrm{x}$ is infected, it recovers), $N^{(x+1, x)}$ and $N^{(x-1, x)}$, with intensity $\lambda$, whose points represent the "infection times" (i.e., times in which, if one of the sites $x+1$ or $x-1$ is infected, the site $x$ becomes infected too). Processes corresponding to different sites are independent. Let us use the notation $\mathbb{Z}^{*}$ for the set of ordered pairs $\{z=(x, y) \in$ $\mathbb{Z} \times \mathbb{Z}:|x-y|=1\}$. In what follows, in order to simplify notations, for a process of the type $N^{(x+1, x)}$ we shall use sometimes the symbol $N^{z}$, where $z=(x+1, x) \in \mathbb{Z}^{*}$. The family of Poisson processes which describe all the possible jump times of the whole system is given by

$$
\left\{N^{x}, N^{z} ; x \in \mathbb{Z}, z \in \mathbb{Z}^{*}\right\} .
$$

Therefore, we take as probability space the path space of these processes, that is, if $(\mathcal{S}, \mathcal{B}(\mathcal{S}))$ is the measurable space of locally finite subsets of $\mathbb{R}$ considered in section 3 , we take

$$
\Omega=\mathcal{S}^{\mathbb{Z}} \times \mathcal{S}^{\mathbb{Z}^{*}},
$$

provided with a metric that induces the product topology and we denote by $\mathcal{F}$ the corresponding Borel $\sigma$-field. We denote by $\mathbb{P}$ the probability on $\mathcal{F}$ induced by the Poisson processes in (4.2). So, each $\omega \in \Omega$ has an infinite number of components, indexed in $\mathbb{Z}$ and $\mathbb{Z}^{*}$, each one of which is an element of $\mathcal{S}$. We can pose $\omega=\left(\omega^{x}, \omega^{z} ; x \in \mathbb{Z}, z \in \mathbb{Z}^{*}\right)$. Now, for $t \in \mathbb{R}$, we define $\theta_{t} \omega$ componentwise on $\Omega$ as follows: $\theta_{t} \omega=\left(\vartheta_{t} \omega^{x}, \vartheta_{t} \omega^{z} ; x \in\right.$ $\left.\mathbb{Z}, z \in \mathbb{Z}^{*}\right)=\left(\omega^{x}-t, \omega^{z}-t ; x \in \mathbb{Z}, z \in \mathbb{Z}^{*}\right)$ where $\vartheta_{t}$ is the shift on $\mathcal{S}$, as it was defined in section 3. Now it easy to see that $\theta_{t}$ is a measure preserving transformation on $\Omega$, so that $\theta:=\left(\Omega, \mathcal{F}, \mathbb{P},\left(\theta_{t}\right)_{t \in \mathbb{R}}\right)$ is a metric dynamical system.

We now need to define $\varphi(t, \omega) \xi$, for each $\omega \in \Omega, t>0$ and for any starting configuration $\xi \in X$. 
Using the terminology of the graphical construction (see [Li1, Li2]), for $s, t \in \mathbb{R}, s<t$, and $x, y \in \mathbb{Z}$, we say there is a path from $(y, s)$ to $(x, t)$ if there is a sequence of times $s=t_{0}<t_{1}<\cdots<t_{n}<t_{n+1}=t$ and a sequence in $\mathbb{Z} y=x_{0}, x_{1}, \ldots, x_{n-1}, x_{n}=x$, such that

- for $i=1, \ldots, n,\left|x_{i-1}-x_{i}\right|=1$ and $t_{i} \in \omega^{\left(x_{i-1}, x_{i}\right)}$, i.e. all $t_{i}$ 's are infection times;

- for $i=0, \ldots, n, \omega^{x_{i}} \cap\left[t_{i}, t_{i+1}\right]=\emptyset$, i.e. $x_{i}$ does not recover before infecting a neighbor.

Then, we define

$(\varphi(t, \omega) \xi)(x)=1 \Leftrightarrow$ there is a path from $(y, 0)$ to $(x, t)$ for some $y \in \mathbb{Z}$ with $\xi(y)=1$.

Below we show that this definition is well posed for almost all $\omega \in \Omega$, i.e., for each fixed $\omega$ in a set of full measure $\mathcal{A}$, there exists $M>0$ such that for every $y \in \mathbb{Z} \backslash[-M, M]$ we cannot find any path from $(y, 0)$ to $(x, t)$.

Note that, for a fixed $x \in \mathbb{Z}$, the value $(\varphi(t, \omega) \xi)(x)$ is determined by the jump times in $\left(\omega^{x} \cup \omega^{(x-1, x)} \cup \omega^{(x+1, x)}\right) \cap(0, t]$ and it changes depending on the values which the current configuration has in $x, x+1$ and $x-1$ at that times. By iterating the above argument, we then need to know, for each $s \in\left(\omega^{x} \cup \omega^{(x-1, x)} \cup \omega^{(x+1, x)}\right) \cap(0, t]$, the values of $(\varphi(s, \omega) \xi)(y)$ for $y$ in a subset $I \subset \mathbb{Z}$ which becomes larger and larger. Let us show that the set $I$ is finite ,i.e., $(\varphi(t, \omega) \xi)(x)$ can be completely determined by considering just a finite number of components of $\omega=\left(\omega^{x}, \omega^{z}: x \in \mathbb{Z}, z \in \mathbb{Z}^{*}\right)$ and the values of the starting configuration $\xi$ in the corresponding sites.

Fix $x \in \mathbb{Z}$ and set $\tau_{0}=t, I_{0}=\{x\}$. Now (convening $\max \emptyset=-\infty$ ) define

$$
\tau_{1}(\omega)=\max \left\{\left(\omega^{x} \cup \omega^{(x-1, x)} \cup \omega^{(x+1, x)}\right) \cap(-\infty, t]\right\} \quad \text { and } \quad I_{1}=\{x-1, x, x+1\} .
$$

Note that $t-\tau_{1}$ has Exponential distribution of mean $1 /(2 \lambda+1)$. Now, if $\tau_{1} \leq 0$, then neither recoveries nor infections have taken place during the time interval $(0, t]$, thus $(\varphi(t, \omega) \xi)(x)=\xi(x)$. Otherwise, suppose we had defined $\left(\varphi\left(\tau_{1}^{-}, \omega\right) \xi\right)(y)$ for $y \in I_{1}$. Then, according to the interpretation of recovery times and infection times, we would let $(\varphi(t, \omega) \xi)(x)=\left(\varphi\left(\tau_{1}, \omega\right) \xi\right)(x)=1$ if and only if one of the following conditions hold:

- $\tau_{1} \notin \omega^{x}$ and $\left(\varphi\left(\tau_{1}^{-}, \omega\right) \xi\right)(x)=1$;

- $\tau_{1} \in \omega^{(x-1, x)},\left(\varphi\left(\tau_{1}^{-}, \omega\right) \xi\right)(x-1)=1$ or $\tau_{1} \in \omega^{(x+1, x)},\left(\varphi\left(\tau_{1}^{-}, \omega\right) \xi\right)(x+1)=1$.

Thus we obtain $(\varphi(t, \omega) \xi)(x)$ from the knowledge of $\left(\varphi\left(\tau_{1}^{-}, \omega\right) \xi\right)(y)$ for $y \in I_{1}$. Now we proceed recursively, letting

$$
I_{n}=\{x-n, x-n+1, \ldots, x, \ldots, x+n-1, x+n\}
$$

and

$$
\tau_{n+1}=\max \left\{\bigcup_{y \in I_{n}}\left(\omega^{y} \cup \omega^{(y-1, y)} \cup \omega^{(y, y+1)}\right) \cap\left(-\infty, \tau_{n}\right)\right\}
$$


By standard properties of Poisson processes, it follows that the random variables $\left(\tau_{n}-\tau_{n+1}\right)_{n \geq 0}$ are independent, and $\tau_{n}-\tau_{n+1}$ has a Exponential distribution with mean $1 /[(2 n+1)(2 \lambda+1)]$. If $\tau_{n}>0$ but $\tau_{n+1} \leq 0$, then we set $\left(\varphi\left(\tau_{n}^{-}, \omega\right) \xi\right)(y)=\xi(y)$ for all $y \in I_{n}$. Otherwise, if $\tau_{n+1}>0$, then $\left(\varphi\left(\tau_{n}^{-}, \omega\right) \xi\right)(y), y \in I_{n}$ can be obtained in terms of $\left(\varphi\left(\tau_{n+1}^{-}, \omega\right) \xi\right)(z), z \in I_{n+1}$ through the updating rule described above.

A sufficient condition for this procedure to end (almost surely) in a finite number of steps, is

$$
\lim _{n \rightarrow+\infty} \tau_{n}=-\infty \text { a.s. }
$$

This is easily proved as follows. First write $\tau_{n}=t-\sum_{k=0}^{n-1}\left(\tau_{k+1}-\tau_{k}\right)$. Thus $t-\tau_{n}$ is the sum of independent, exponential random variables. This allows to compute $\mathbb{E}\left(e^{-\tau_{n}}\right)$, and show that these expectations tend to zero as $n \rightarrow+\infty$, from which (4.3) follows.

Now, let $\mathcal{A}_{x, t}$ the set of those $\omega \in \Omega$ such that the above construction ends in a finite number of steps. We just saw that $\mathbb{P}\left(\mathcal{A}_{x, t}\right)=1$. Note that this set depends on $x$ and $t$, but does not depend on $\xi$. If we let $\mathcal{A}_{t}=\bigcap_{x \in \mathbb{Z}} \mathcal{A}_{x, t}$, we have that, for $\omega \in \mathcal{A}_{t}$, $y \in \mathbb{Z}$ and $0 \leq s \leq s+h \leq t$, the value of $\varphi\left(h, \theta_{s} \omega\right) \xi(y)$ can be constructed in a finite number of steps. In other words, $\theta_{s} \mathcal{A}_{h} \subseteq \mathcal{A}_{t}$. Therefore, letting

$$
\mathcal{A}=\cap_{N \in \mathbb{Z}} \theta_{N} \mathcal{A}_{N}
$$

$\mathcal{A}$ has full measures, $\theta_{t} \mathcal{A}=\mathcal{A}$ for every $t \in \mathbb{R}$, and for $\omega \in \mathcal{A}, \varphi\left(t, \theta_{s} \omega\right)$ is well defined for every $t \geq 0$ and $s \in \mathbb{R}$. For $\omega \notin \mathcal{A}$, we are free to define $\varphi(t, \omega)=\mathrm{id}_{X}$. Note that, according to the definition of Random Dynamical System, $\varphi$ must be defined everywhere. The fact that $\theta_{t} \mathcal{A}=\mathcal{A}$ for every $t \in \mathbb{R}$, guarantees that the cocycle property holds everywhere (see Proposition 6 below).

Proposition 6 . The map $\varphi$ constructed above defines a continuous, monotone Random Dynamical System over $\theta$.

Proof. We first prove measurability of $\varphi$. Let $\omega \in \Omega$ and, for $N \in \mathbb{Z}^{+}$, let $\Pi_{N}(\omega) \in \Omega$ be defined as follows:

$$
\Pi_{N}(\omega)^{x}= \begin{cases}\omega^{x} & \text { for }|x| \leq N \\ \emptyset & \text { otherwise }\end{cases}
$$

and, for $|x-y|=1$

$$
\Pi_{N}(\omega)^{(x, y)}= \begin{cases}\omega^{(x, y)} & \text { for }|x| \leq N,|y| \leq N \\ \emptyset & \text { otherwise. }\end{cases}
$$

In other words $\Pi_{N}(\omega)$ contains only those jump times of $\omega$ that involve sites in $\{-N, \ldots, N\}$. Define, now

$$
\varphi_{N}(t, \omega) \xi:=\varphi\left(t, \Pi_{N}(\omega)\right) \xi
$$


Note that $\varphi_{N}(t, \omega) \xi(x)=\xi(x)$ for $|x|>N$ and $t>0$. Now let $\sigma_{0}=0, \sigma_{1}, \sigma_{2}, \ldots, \sigma_{n}, \ldots$ be an increasing enumeration of the positive jump times in $\Pi_{N}(\omega)$, i.e. of the set

$$
\bigcup_{\substack{|x| \leq N,|y| \leq N \\|x-y|=1}}\left(\omega^{x} \cup \omega^{(x, y)}\right) .
$$

Note that the $\sigma_{n}$ are random variables, and, for $n \geq 1$ and $\omega$ fixed, $\varphi_{N}\left(\sigma_{n}, \omega\right) \xi$ is obtained from $\xi$ by modifying finitely many components. In other words, $\varphi_{N}\left(\sigma_{n}, \omega\right) \xi$ can be written as a finite sum of the form

$$
\sum_{i} \mathbf{1}_{A_{i}}(\omega) \xi^{V_{i}}
$$

In (4.4) each $A_{i}$ is a set of the form $\left\{\omega: \sigma_{1} \in \omega^{z_{1}}, \sigma_{2} \in \omega^{z_{2}}, \ldots, \sigma_{n} \in \omega^{z_{n}}\right\}$, where $z_{j} \in \mathbb{Z}$ or $\mathbb{Z}^{*}$ and $\xi^{V_{i}}$ is obtained from $\xi$ by changing the coordinates (corresponding to the realization of the event $A_{i}$ ) indexed by the elements of $V_{i}$. In particular, it follows that the $A_{i}$ 's are measurable, and, by $(4.4), \varphi_{N}\left(\sigma_{n}, \omega\right) \xi$ is jointly measurable in $\omega, \xi$. Finally, since

$$
\varphi_{N}(t, \omega) \xi=\sum_{n \geq 0} \varphi_{N}\left(\sigma_{n}, \omega\right) \xi \mathbf{1}_{\left[\sigma_{n}, \sigma_{n+1}\right)}(t)
$$

we have that $\varphi_{N}(t, \omega) \xi$ is jointly measurable in all variables. By the graphical construction described above, we have that, for all $\omega \in \Omega$

$$
\varphi(t, \omega) \xi=\lim _{N \rightarrow+\infty} \varphi_{N}(t, \omega) \xi
$$

and measurability of $\varphi$ follows. Continuity of $\varphi$ follows from the observation that, for each $\omega \in \Omega$ and $x \in \mathbb{Z}, \varphi(t, \omega) \xi(x)$ depends on finitely many components of $\xi$. Monotonicity is immediate form the characterization of the dynamics in terms of existence of paths. We are so left to show that the cocycle property holds for $\varphi$. We need to show that, for each $x \in X$,

$$
(\varphi(t+s, \omega) \xi)(x)=1 \Leftrightarrow\left(\varphi\left(t, \theta_{s} \omega\right) \varphi(s, \omega) \xi\right)(x)=1
$$

Indeed,if $(\varphi(t+s, \omega) \xi)(x)=1$ then, for some $y \in \mathbb{Z}$ such that $\xi(y)=1$, we can find $t_{0}=0<t_{1}<\ldots<t_{m}<t_{m+1}=t+s$ and $x_{0}=y, x_{1}, \ldots, x_{m}=x$ such that they form a path from $(y, 0)$ to $(x, t+s)$. This implies that there exists $k \in\{0, \ldots, m+1\}$ such that $s \in\left[t_{k}, t_{k+1}\right)$ and there is a path from $(y, 0)$ to $\left(x_{k}, s\right)$, i.e. $(\varphi(s, \omega) \xi)\left(x_{k}\right)=1$. But $\left(x_{k}, s\right),\left(x_{k+1}, t_{k+1}\right), \ldots,\left(x_{m}, t_{m}\right),(x, t+s)$ is a path from $\left(x_{k}, s\right)$ to $(x, t+s)$ and so $\left(\varphi\left(t, \theta_{s} \omega\right) \varphi(s, \omega) \xi\right)(x)=1$. On the other hand, suppose that there exists a path $\gamma_{1}$ from $(y, s)$ to $(x, t+s)$ for some $y$ such that $(\varphi(s, \omega) \xi)(y)=1$ i.e. for some $y$ such that there is a path $\gamma_{2}$ from $(z, 0)$ to $(y, s)$, where $\xi(z)=1$. Then $\gamma_{1} \cup \gamma_{2}$ is a path from $(z, 0)$ to $(x, t+s)$ and so $(\varphi(t+s, \omega) \xi)(x)=1$ 
Remark 2 It is easy to see that the map $\varphi_{N}$ in the proof of Proposition 6 defines a continuous monotone random dynamical system over $\theta$. The cocycle property follows from the cocycle property of $\varphi$ and from the fact that, for each $N \geq 0$, we have $\theta_{s} \Pi_{N}(\omega)=\Pi_{N}\left(\theta_{s} \omega\right)$ for all $\omega \in \Omega$ and $s \in \mathbb{R}$.

As we shall see in the proof of the Proposition below, the one-point motions of $\varphi_{N}$ can be seen as finite state Contact processes.

Proposition 7 . The map $\varphi$ has independent increments. In particular, the one-point motions of $\varphi$, i.e. the stochastic processes $\left(\{\varphi(t, \cdot) \xi\}_{t \in \mathbb{R}^{+}} ; \xi \in X\right)$ are homogeneous Markov processes. Moreover, the associated Markov semigroup $\left(S_{t}\right)_{t \in \mathbb{R}^{+}}$has the Feller property and its generator is the operator A defined in (4.1).

Proof. For each fixed $\xi \in X$, from the construction of $\varphi$ it follows that, for every $t \in \mathbb{R}^{+}$ and $s \in \mathbb{R}$, the random variables $\varphi\left(t, \theta_{s} \cdot\right) \xi$ and $\varphi_{N}\left(t, \theta_{s} \cdot\right) \xi$ are measurable with respect to the $\sigma$-algebra generated by $\left\{\left|\omega^{x} \cap[s, w]\right|,\left|\omega^{z} \cap[s, w]\right| ; x \in \mathbb{Z}, z \in \mathbb{Z}^{*}, s \leq w \leq t\right\}$, which we denote by $\mathcal{F}_{s, t}$. Moreover, from the independence of increments of Poisson processes we have that, for all $s<t \leq u<v, \mathcal{F}_{s, t}$ is independent of $\mathcal{F}_{u, v}$.

Now, let us take $t_{1}, \ldots, t_{n}$ in $\mathbb{R}$ with $t_{1}<\ldots<t_{n}$ and consider the random variables

$$
\varphi\left(t_{2}-t_{1}, \theta_{t_{1}} \cdot\right), \ldots, \varphi\left(t_{n}-t_{n-1}, \theta_{t_{n-1}} \cdot\right) .
$$

These are random variables with values in the set $\mathbb{G}$ of functions from $X$ to itself, provided with the $\sigma$-algebra which makes the evaluation mappings $\Phi_{\eta}: f \mapsto f(\eta)$ measurable. A system of generators for this $\sigma$-algebra is the set $\left\{\Phi_{\eta}^{-1}(B) ; \eta \in X, B \in\right.$ $\mathcal{B}(X)\}$. Then, if we take, for $i=1, \ldots, n-1, \Phi_{\eta_{i}}^{-1}\left(B_{i}\right)$ with $\eta_{i} \in X$ and $B_{i} \in \mathcal{B}(X)$, we have,

$$
\begin{aligned}
\mathbb{P}\left(\bigcap_{i=1}^{n-1}\left\{\varphi\left(t_{i+1}-t_{i}, \theta_{t_{i}} \cdot\right) \in \Phi_{\eta_{i}}^{-1}\left(B_{i}\right)\right\}\right) & =\mathbb{P}\left(\bigcap_{i=1}^{n-1}\left\{\varphi\left(t_{i+1}-t_{i}, \theta_{t_{i}} \cdot\right) \eta_{i} \in B_{i}\right\}\right) \\
& =\prod_{i=1}^{n-1} \mathbb{P}\left\{\varphi\left(t_{i+1}-t_{i}, \theta_{t_{i}} \cdot\right) \eta_{i} \in B_{i}\right\} \\
& =\prod_{i=1}^{n-1} \mathbb{P}\left\{\varphi\left(t_{i+1}-t_{i}, \theta_{t_{i}} \cdot\right) \in \Phi_{\eta_{i}}^{-1}\left(B_{i}\right)\right\}
\end{aligned}
$$

The same property holds for $\varphi_{N}$. Then, from proposition 1, it follows that, for each $\xi \in X,\{\varphi(t, \cdot) \xi\}_{t \geq 0}$ and $\left\{\varphi_{N}(t, \cdot) \xi\right\}_{t \geq 0}$ are homogeneous Markov processes.

Now we show that the operator $A$ defined by (4.1) is the generator associated with $\varphi$. Denote by $\left(S_{t}\right)_{t \geq 0}$ the Markov semigroup associated with $\varphi$, i.e.

$$
S_{t} f(\xi)=\mathbb{E}[f(\varphi(t, \cdot) \xi)],
$$

for $f \in \mathrm{C}(X)$. By Dominated Convergence Theorem

$$
S_{t} f(\xi)=\lim _{N \rightarrow+\infty} S_{t}^{N} f(\xi):=\lim _{N \rightarrow+\infty} \mathbb{E}\left[f\left(\varphi_{N}(t, \omega) \xi\right)\right] .
$$


Moreover, an easy exercise on Poisson processes shows that, for $t \geq 0, S_{t}^{N}=e^{t A_{N}}$, where

$$
A_{N} f(\xi)=\sum_{\substack{x \in \mathbb{Z}^{d} \\|x| \leq N}} c(x, \eta)\left[f\left(\xi^{x}\right)-f(\xi)\right] .
$$

Now let $\left(\bar{S}_{t}\right)_{t \geq 0}$ be the Markov semigroup associated to $A$, i.e., $\bar{S}_{t}=e^{t A}$ for $t \geq 0$. If $f \in \mathcal{D}(X)$, it is immediately seen that

$$
A f=\lim _{N \rightarrow+\infty} A_{N} f
$$

Then (by Theorem 2.12, p.17 of [Li1]) we have $\lim _{N \rightarrow+\infty} S_{t}^{N} f=\bar{S}_{t} f$ for all $f \in \mathcal{C}(X)$ and, by (4.5), it follows that $S_{t}=\bar{S}_{t}=e^{t A}$ for all $t \geq 0$.

We can now apply to $\varphi$ the results of Section 2. The $\omega$-limit set of the state space $\Omega_{X}$ is a $\mathcal{B}$-attractor for every $\mathcal{B}$. Moreover, the system has two equilibrium points, $\sigma^{-}:=\lim _{t \rightarrow+\infty} \varphi\left(t, \theta_{-t^{*}}\right) \overline{0}=\overline{0}$ and $\sigma^{+}:=\lim _{t \rightarrow+\infty} \varphi\left(t, \theta_{-t^{*}}\right) \overline{1}$ and we have, for all $\omega \in \Omega$

$$
\Omega_{X}(\omega) \subset\left[\overline{0}, \sigma^{+}(\omega)\right]:=\left\{\eta \in X: \overline{0} \leq \eta \leq \sigma^{+}(\omega)\right\}
$$

Note that the relation above holds for every monotone RDS which realizes the contact process (but it is not true in general, since, if $\varphi$ is not monotone, the existence of $\sigma^{+}$is not guaranteed).

Clearly, if $\lambda \leq \lambda_{c}$ we have, $\sigma^{+}=\overline{0} \mathbb{P}$-almost surely. Indeed, the law of $\varphi\left(t, \theta_{-t} \cdot\right) \overline{1}$ is given by $\delta_{\overline{1}} S_{t}$ which in this case converges to $\delta_{\overline{0}}$. As a consequence, if $\lambda \leq \lambda_{c}$, we have $\Omega_{X}=\{\overline{0}\} \mathbb{P}$-almost surely. The interesting case is when $\lambda>\lambda_{c}$.

A first result about the pull-back behavior of $\varphi$ is the following.

Theorem 2 . Suppose $\lambda>\lambda_{c}$.

1. Let $\xi$ be a local configuration, i.e. $\sum_{x} \xi(x)<+\infty$, and let

$$
\sigma^{+}(\omega)=\lim _{t \rightarrow+\infty} \varphi\left(t, \theta_{-t} \omega\right) \overline{1}
$$

Then

$$
\lim _{t \rightarrow+\infty} d\left(\varphi\left(t, \theta_{-t} \cdot\right) \xi,\left\{\overline{0}, \sigma^{+}\right\}\right)=0 \quad \mathbb{P} \text {-a.s. }
$$

2. If $\xi$ is such that $\sum_{x} \xi(x)=+\infty$, then

$$
\lim _{t \rightarrow+\infty} \varphi\left(t, \theta_{-t} \cdot\right) \xi=\sigma^{+}(\omega)
$$

in probability. 
Proof. Define the configuration $\eta^{-}$by

$$
\eta^{-}(x):=\left\{\begin{array}{ll}
1 & \text { for } x \leq 0 \\
0 & \text { for } x>0
\end{array},\right.
$$

$\eta^{+}(x):=\eta^{-}(-x)$, and

$$
\begin{aligned}
& r_{t}(\omega)=\max \left\{x: \varphi(t, \omega) \eta^{-}(x)=1\right\} \\
& l_{t}(\omega)=\min \left\{x: \varphi(t, \omega) \eta^{+}(x)=1\right\} .
\end{aligned}
$$

The key step in the proof of Theorem 1 is to show that, for $\lambda>\lambda_{c}, r_{t} \rightarrow+\infty$ and $l_{t} \rightarrow-\infty$ a.s. as $t \rightarrow+\infty$. For proving Theorem 2 we need to show, instead, that

$$
\lim _{t \rightarrow+\infty} r_{t} \circ \theta_{-t}=+\infty \quad \text { a.s. }
$$

To prove (4.10) we use self-duality of the Contact process. We consider a new random dynamical system $\hat{\varphi}$ defined as follows: for $t \in \mathbb{R}$ let $\hat{\theta}_{t}$ the map on $\Omega$ given by $\hat{\theta}_{t} \omega=\theta_{-t} \omega$, where $\theta_{t}$ is the componentwise shift involved in the definition of $\varphi$. Clearly, $\hat{\theta}:=\left(\Omega, \mathcal{F}, \mathbb{P},\left(\hat{\theta}_{t}\right)_{t \in \mathbb{R}}\right)$ is a metric dynamical system. The map $\hat{\varphi}$ is defined with the same procedure as $\varphi$, but reversing the direction of time. For $\omega \in \mathcal{A}$, we set $\hat{\varphi}(t, \omega) \xi(x)=1$ if and only if there is a path from $(x,-t)$ to $(y, 0)$, for some $y \in \mathbb{Z}$ with $\xi(y)=1$; for $\omega \notin \mathcal{A}$ we set $\hat{\varphi}(t, \omega) \xi \equiv \xi$. Now, with the same arguments used for $\varphi$, it can be verified that $\hat{\varphi}$ defines a random dynamical system over $\hat{\theta}$. Since both $\mathcal{A}$ and the probability $\mathbb{P}$ are invariant for time reversal, it follows that the two processes $(\varphi(t, \cdot) \xi)_{t \geq 0}$ and $(\hat{\varphi}(t, \cdot) \xi)_{t \geq 0}$ have the same law, so they are both Contact processes with parameter $\lambda$.

Note now that the statement $\lim _{t \rightarrow+\infty} r_{t}\left(\theta_{-t} \omega\right)=+\infty$ is equivalent to the fact that, for every $M>0$ there exists $t_{M}>0$ such that for every $t>t_{M}$ there are $x \leq 0$ and $y \geq M$ and a path from $(-t, x)$ to $(0, y)$. By translation invariance of $\mathbb{P}$, this last event has the same probability of: for every $M>0$ there exists $t_{M}>0$ such that for every $t>t_{M}$ there are $x \leq-M$ and $y \geq 0$ and a path from $(-t, x)$ to $(0, y)$, i.e.

$$
\lim _{t \rightarrow+\infty} \hat{l}_{t}=-\infty
$$

where

$$
\hat{l}_{t}(\omega)=\min \left\{x: \hat{\varphi}(t, \omega) \eta^{+}(x)=1\right\} .
$$

Since $\hat{\varphi}(t, \omega) \eta$ is a supercritical Contact process, (4.11) holds true with probability one, and this establishes (4.10).

By symmetry, we also have

$$
\lim _{t \rightarrow+\infty} l_{t} \circ \theta_{-t}=-\infty \quad \text { a.s. }
$$

At this point we use two well known properties of the graphical construction of the Contact process. The first is the additivity: for every $\xi, \eta \in X$ and $\omega \in \Omega$

$$
\varphi(t, \omega)(\xi \vee \eta)=\varphi(t, \omega) \xi \vee \varphi(t, \omega) \eta
$$


The second reads as follows. Let $\eta^{0}$ be defined by $\eta^{0}(0)=1$ and $\eta^{0}(x)=0$ for every $x \neq 0$. Then, for all $\omega \in \Omega$

$$
\text { either } \varphi(t, \omega) \eta^{0}=\overline{0} \quad \text { or } \varphi(t, \omega) \eta^{0}(x)=\varphi(t, \omega) \overline{1}(x) \quad \forall x \in\left[l_{t}(\omega), r_{t}(\omega)\right] .
$$

At this point we are ready to prove statement 1. of the Theorem. For $\xi=\eta^{0},(4.6)$ follows easily from (4.10), (4.12) and (4.14). By translation invariance, we get (4.6) for $\xi=\eta^{x}, x \in \mathbb{Z}^{d}$, where $\eta^{x}$ is defined by $\eta^{x}(x)=1$ and $\eta^{x}(y)=0$ otherwise. If $\xi$ is a local configuration, then it can be written in the form $\xi=\eta^{x_{1}} \vee \eta^{x_{2}} \vee \cdots \vee \eta^{x_{m}}$. Thus (4.6) follows from (4.13).

We now prove statement 2. Let's use the notations $Y_{t}$ for $\varphi\left(t, \theta_{-t} \cdot\right) \overline{1}$ and $X_{t}$ for $\varphi\left(t, \theta_{-t}\right) \xi$. For $t \geq 0, X_{t}$ and $Y_{t}$ are random variables with values in $X=\{0,1\}^{\mathbb{Z}}$ and we know that $X_{t} \leq Y_{t}$ for every $t, \lim _{t} Y_{t}=\sigma^{+} \mathbb{P}$-a.s. and $\lim _{t} X_{t}=\sigma^{+}$in distribution (Theorem 1). If $\xi \in X$, let us consider the function

$$
f(\xi)=\sum_{x \in \mathbb{Z}} \frac{1}{2^{|x|}} \xi(x)
$$

which is a bounded continuous increasing function (note that $f(\xi)=d(\xi, \overline{0})$ where $d$ is the usual distance in $X)$. Now we want to proof that $d\left(Y_{t}, X_{t}\right) \longrightarrow 0$ in probability. Suppose that this is not true: then we can find $\varepsilon, \delta>0$ and a sequence of times $\left(t_{k}\right)_{k}$ such that

$$
\mathbb{P}\left(d\left(Y_{t_{k}}, X_{t_{k}}\right)>\varepsilon\right)>\delta \text { for all } k \geq 0 .
$$

Since $X_{t} \leq Y_{t}$ for all $t, d\left(Y_{t_{k}}, X_{t_{k}}\right)=f\left(Y_{t_{k}}\right)-f\left(X_{t_{k}}\right)$. Then, for every $k \geq 0$

$$
\begin{aligned}
\mathbb{E}\left[f\left(Y_{t_{k}}\right)-f\left(X_{t_{k}}\right)\right] & \geq \varepsilon \mathbb{P}\left(d\left(Y_{t_{k}}, X_{t_{k}}\right)>\varepsilon\right) \\
& >\varepsilon \delta .
\end{aligned}
$$

But the first quantity of the inequality above should converge to zero, because both the sequences $\left(Y_{t_{k}}\right)_{k}$ and $\left(X_{t_{k}}\right)_{k}$ converge to $\sigma^{+}$in distribution. So, we have a contradiction and the proof is complete.

A local set $B \subset X$ is a set of the form $B=\{\eta \in X: \eta(x)=0 \forall x \notin \Lambda\}$, where $\Lambda$ is a given finite subset of $\mathbb{Z}$.

Corollary 2 . Let $\varphi$ the $R D S$ defined above and let $\mathcal{B}$ be the family of all the local subsets of $X$. Then the random set $\left\{\overline{0}, \sigma^{+}\right\}$is a $\mathcal{B}$-random attractor for $\varphi$. Moreover, for each local configuration $\xi \neq \overline{0}$ we have

$$
\Omega_{\{\xi\}}(\omega)=\left\{\overline{0}, \sigma^{+}(\omega)\right\} \text { for } \mathbb{P} \text {-almost all } \omega
$$

Proof. The first assertion is an immediate consequence of Theorem 2. For the second, we have to prove that, for a local configuration $\xi$, the pull-back trajectory starting at $\xi$ has exactly two limit points, $\overline{0}$ and $\sigma^{+}(\omega)$. We first observe that, for $t>h>0$ fixed, the event $\left\{\varphi\left(h, \theta_{-t} \omega\right) \xi=\overline{0}\right\}$ has a probability which is strictly positive and 
independent of $t$. Moreover, $\varphi\left(h, \theta_{-t} \omega\right) \xi=\overline{0} \Rightarrow \varphi\left(t, \theta_{-t} \omega\right) \xi=\overline{0}$. Thus, by the fact that Poisson processes have independent increments and Borel-Cantelli Lemma, for $\mathbb{P}$-almost all $\omega$ we can find a sequence $\left\{t_{n}\right\}_{n \geq 0}$ with $t_{n} \uparrow+\infty$ and $\varphi\left(t_{n}, \theta_{-t_{n}} \omega\right) \xi=\overline{0}$ for all $n \geq 0$. Therefore $\overline{0} \in \Omega_{\{\xi\}}(\omega)$. Now we want to show that $\sigma^{+} \in \Omega_{\{\xi\}} \mathbb{P}_{-}$ almost surely. By monotonicity, we can assume $\xi=\eta^{0}$. Since (4.14) holds, the proof will be complete if we show that, for $\mathbb{P}$-almost all $\omega$, we can find a sequence $\left\{s_{n}\right\}_{n \geq 0}$ with $s_{n} \uparrow+\infty$ such that $\varphi\left(s_{n}, \theta_{-s_{n}} \omega\right) \xi \neq \overline{0}$ for all $n \geq 0$. If we write the condition above in terms of the dual $\operatorname{RDS} \hat{\varphi}$, then we have to show that the event $\left\{\left(\hat{\varphi}\left(s_{n}, \cdot\right) \overline{1}\right)(0)=1 \forall n \geq 0\right.$ for some $\left.s_{n} \uparrow+\infty\right\}$ has probability 1 . This is a well known fact which is proved in Theorem 2.27 of [Li2] in the general case $X=\{0,1\}^{\mathbb{Z}^{d}}$ (indeed in the case $d=1$ admits a much simpler proof).

Remark 3 . Identity (4.15) shows that, for $\mathbb{P}$-almost all $\omega$, for any local configuration $\xi$ and any $V \subset \mathbb{Z}$ finite, there exists a time $T(\omega)$ such that, for all $t>T(\omega)$, either $\left(\varphi\left(t, \theta_{-t} \omega\right) \xi\right)(x)=0 \forall x \in V$ or $\left(\varphi\left(t, \theta_{-t} \omega\right) \xi\right)(x)=\sigma^{+}(\omega)(x) \forall x \in V$, and this second possibility occurs for $t$ arbitrarily large. Therefore any "finite portion" of $\sigma^{+}$can be perfectly simulated in a finite random time starting from a local configuration and hence simulation involves only a finite number of updatings.

Remark 4 . The proof of Theorem 2 relies on relatively few properties of the Contact process, namely, (4.10), (4.12), (4.13) and (4.14). The crucial point is the proof of (4.10) and (4.12), that here relies on self-duality. It is not hard to exhibit non self-dual processes for which (4.13) and (4.14) hold. For example, we may consider the discretetime process defined as follows. In the lattice $\mathbb{Z}^{2}$ and $(x, n) \in \mathbb{Z}^{2}$, consider the oriented bonds $(x, n) \rightarrow(x, n+1),(x, n) \rightarrow(x-1, n+1),(x, n) \rightarrow(x+1, n+1)$. The bond $(x, n) \rightarrow(x, n+1)$ is open with probability $p \in(0,1)$. If $(x, n) \rightarrow(x, n+1)$ is closed, then also the other two bonds are closed. Conditionally to $(x, n) \rightarrow(x, n+1)$ being open, then each one of the two bonds $(x, n) \rightarrow(x-1, n+1),(x, n) \rightarrow(x+1, n+1)$ is open with probability $q \in(0,1)$, independently of each other. Bonds departing at different $(x, n)$ behave independently. In this context, a path is a sequence of adjacent open bonds. We denote by $\omega$ a configuration of all such bonds. We may define a random dynamical system by $\varphi(n, \omega) \xi(x)=1$ if and only if there is a path from $(y, 0)$ to $(x, n)$. This process has supercritical behavior for $p$ and $q$ large enough, i.e. $\lim _{n \rightarrow+\infty} \varphi\left(n, \theta_{-n} \omega\right) \overline{1} \neq \overline{0}$. This can be seen, for instance, by comparison with oriented percolation (see $[\mathrm{Du}]$ ). This process is not self-dual, but properties (4.10) and (4.12) can be proved directly, via a modified version of the Liggett Subadditive Ergodic Theorem (see [Li1]). We refer to [Mi] for details. Thus, the conclusions of Theorem 2 and Corollary 2 hold also in this case.

Unfortunately, we are still not able to give a complete description of $\Omega_{X}$. The main problem is that in this case, since we are not restricted to local subsets of the state space, when we consider pull-back trajectories, we can change their starting point an infinite number of times.

Below we show that the problem of describing $\Omega_{X}$ for the Contact process can be reformulated in such a way that it becomes quite similar to what we saw in section 3 for 
random walk. In the proof of Proposition 5 it was crucial the (almost sure) coalescence of the two symmetric random walks $Z^{1}$ and $Z^{2}$ after a finite time. In Proposition 8 , we show that a sufficient condition for having $\Omega_{X}=\left\{\overline{0}, \sigma^{+}\right\}$is the coalescence of the stochastic processes $\left\{l_{t}^{x}\right\}_{t \geq 0}$ and $\left\{l_{t}^{y}\right\}_{t \geq 0}$ (see below for the definition).

We do not have good control of the joint law of these processes, so we have not succeeded in proving that coalescence occurs with probability one.

We shall use the following notations: if $B$ is a subset of $\mathbb{Z}, \eta^{B} \in X$ is the configuration given by $\eta^{B}(y)=1$ if $y \in B$ and $\eta^{B}(y)=0$ if $y \notin B$ (if $B=\{x\}$ we use the abbreviate notation $\left.\eta^{x}\right)$. Then we let $r_{t}^{x}(\omega):=\max \left\{z: \varphi(t, \omega) \eta^{(-\infty, x]}(z)=1\right\}$ and $l_{t}^{x}(\omega):=\min \left\{z: \varphi(t, \omega) \eta^{[x,+\infty)}(z)=1\right\}$.

We shall denote by $\hat{r}_{t}^{x}$ and $\hat{l}_{t}^{x}$ the analogous quantities for the dual RDS $\hat{\varphi}$.

Proposition 8 . Suppose that the following conjecture holds:

$$
\lim _{t \rightarrow+\infty}\left|\hat{l}_{t}^{y}-\hat{l}_{t}^{x}\right|=0 \quad \mathbb{P} \text {-a.s. for all } x, y \in \mathbb{Z}
$$

Then we have $\Omega_{X}(\omega)=\left\{\overline{0}, \sigma^{+}(\omega)\right\} \quad \mathbb{P}$-a.s.

Proof. Clearly, if (4.16) holds, by symmetry it holds also for $\hat{r}_{t}^{x}$ and $\hat{r}_{t}^{y}$ and we can assume that this property holds true for all $\omega$. For simplicity, we shall also suppose that for all $\omega$ we have $\lim _{t} r_{t}^{x}\left(\theta_{-t} \omega\right)=+\infty$ and $\lim _{t} l_{t}^{x}\left(\theta_{-t} \omega\right)=-\infty$ for each $x \in \mathbb{Z}$.

Let us fix $\omega$ and take $\zeta=\zeta(\omega) \in \Omega_{X}(\omega)$. We shall prove that, if $\zeta \neq \overline{0}$, then $\zeta=\sigma^{+}(\omega)$. Since $\zeta \in \Omega_{X}(\omega)$, from the definition of attractor it follows that there is a sequence of configurations $\left\{\chi_{n}\right\}_{n \geq 0}$ and a sequence of times $\left\{t_{n}\right\}_{n \geq 0}$, with $\lim _{n} t_{n}=+\infty$, such that

$$
\lim _{n \rightarrow+\infty} \varphi\left(t_{n}, \theta_{-t_{n}} \omega\right) \chi_{n}=\zeta .
$$

Now, if $\zeta \neq \overline{0}$, there is $x \in \mathbb{Z}$ such that $\zeta(x)=1$ and then

$$
\lim _{n \rightarrow+\infty} \varphi\left(t_{n}, \theta_{-t_{n}} \omega\right) \chi_{n}(x)=1 .
$$

This means that there is $\bar{n} \geq 0$ such that, for $n \geq \bar{n}$, we can always find a path from $\left(y_{n},-t_{n}\right)$ to $(x, 0)$ for some $y_{n} \in \mathbb{Z}$ such that $\chi_{n}\left(y_{n}\right)=1$. Now consider the sequence $\left\{\eta^{y_{n}}\right\}_{n \geq \bar{n}}$ : note that, for each $n \geq \bar{n}, \varphi\left(t_{n}, \theta_{-t_{n}} \omega\right) \eta^{y_{n}}(x)=1$ and $\xi^{y_{n}} \leq \chi_{n}$.

Suppose that there exists a subsequence $\left\{y_{n_{k}}\right\}_{k \geq 0}$ such that $y_{n_{k}}=y$ for $k \geq \bar{k}$. Then, since $\varphi\left(t_{n_{k}}, \theta_{-t_{n_{k}}} \omega\right) \eta^{y_{n_{k}}}=\varphi\left(t_{n_{k}}, \theta_{-t_{n_{k}}} \omega\right) \eta^{y} \neq \overline{0}$ for all $k \geq \bar{k}$ from Theorem 2 it follows that $\lim _{k} \varphi\left(t_{n_{k}}, \theta_{-t_{n_{k}}} \omega\right) \eta^{y_{n_{k}}}=\sigma^{+}(\omega)$.

From this fact, the monotonicity of $\varphi$ and (4.17) it follows that, if $\left\{y_{n}\right\}_{n \geq 0}$ has a constant subsequence, then $\zeta=\sigma^{+}(\omega)$.

In the case where $\left\{y_{n}\right\}_{n \geq 0}$ does not have a constant subsequence, necessarily $\lim _{n}\left|y_{n}\right|=$ $+\infty$. Suppose there is a subsequence $\left\{y_{n_{k}}\right\}_{k \geq 0}$ such that $\lim _{k} y_{n_{k}}=-\infty$ (if $\lim _{k} y_{n_{k}}=$ $+\infty$ we can use a symmetric argument). We begin by proving that $\zeta(z)=\sigma^{+}(\omega)(z)$ 
for each $z \leq x$. Indeed, we know that, for each $k$ big enough, there is a path $\gamma_{1}$ from $\left(y_{n_{k}},-t_{n_{k}}\right)$ to $(x, 0)$ and $y_{n_{k}}<x$. Moreover, since we have $\lim _{t \rightarrow+\infty} l_{t}^{x}\left(\theta_{-t} \omega\right)=-\infty$, then for each $k$ sufficiently big, for some $z \geq x$, there is a path $\gamma_{2}$ from $\left(z,-t_{n_{k}}\right)$ to $\left(l_{t_{n_{k}}}^{x}\left(\theta_{-t_{n_{k}}} \omega\right), 0\right)$ and necessarily $l_{t_{n_{k}}}^{x}\left(\theta_{-t_{n_{k}}} \omega\right)<x$. Then, $\gamma_{1}$ and $\gamma_{2}$ have to cross, and this implies that for all $k$ big enough there is a path from $\left(y_{n_{k}},-t_{n_{k}}\right)$ to $\left(l_{t_{n_{k}}}^{x}\left(\theta_{-t_{n_{k}}} \omega\right), 0\right)$ and a path from $\left(y_{n_{k}},-t_{n_{k}}\right)$ to $(x, 0)$. Then we have

$$
\left[l_{t_{n_{k}}}^{x}\left(\theta_{-t_{n_{k}}} \omega\right), x\right] \subset\left[l_{t_{n_{k}}}^{y_{n_{k}}}\left(\theta_{-t_{n_{k}}} \omega\right), r_{t_{n_{k}}}^{y_{n_{k}}}\left(\theta_{-t_{n_{k}}} \omega\right)\right]
$$

and by (4.14) (which is a property invariant for space translations) we obtain

$$
\varphi\left(t_{n_{k}}, \theta_{-t_{n_{k}}} \omega\right) \eta^{y_{n_{k}}}(z)=\varphi\left(t_{n_{k}}, \theta_{-t_{n_{k}}} \omega\right) \overline{1}(z) \text { for all } z \in\left[l_{t_{n_{k}}}^{x}\left(\theta_{-t_{n_{k}}} \omega\right), x\right] .
$$

Moreover, by monotonicity of $\varphi$

$$
\varphi\left(t_{n_{k}}, \theta_{-t_{n_{k}}} \omega\right) \chi_{n_{k}}(z)=\varphi\left(t_{n_{k}}, \theta_{-t_{n_{k}}} \omega\right) \overline{1}(z) \text { for all } z \in\left[l_{t_{n_{k}}}^{x}\left(\theta_{-t_{n_{k}}} \omega\right), x\right]
$$

Finally, by taking the limit for $k \rightarrow+\infty$ in the relation above, we obtain $\zeta(z)=$ $\sigma^{+}(\omega)(z)$ for all $z \in(-\infty, x]$.

Now, the proof will be complete if we show that $\sup \{x: \zeta(x)=1\}=+\infty$. This is the point where we use conjecture (4.16). Note that, since $\varphi\left(t_{n}, \theta_{-t_{n}} \omega\right) \chi_{n}(x)=1$ for all $n \geq \bar{n}$, we have, $\hat{\varphi}(t, \omega) \eta^{x} \neq \overline{0}$ for all $t \geq 0$, where $\hat{\varphi}$ is the dual RDS. This and (4.14) imply that $\hat{l}_{t}^{x}(\omega)=\min \left\{z: \hat{\varphi}(t, \omega) \eta^{x}(z)=1\right\}$ (remember that $\hat{l}_{t}^{x}$ is the left edge at time $t$ of the dual process starting from configuration $\left.\eta^{[x,+\infty)}\right)$, i.e., for all $t$ there is a path from $\left(\hat{l}_{t}^{x}(\omega),-t\right)$ to $(x, 0)$.

Let us take $y>x$ and consider $\bar{t}=\inf \left\{t: \hat{l}_{t}^{x}(\omega)=\hat{l}_{t}^{y}(\omega)\right\}$. Then there are two paths departing from $\left(\hat{l}_{\bar{t}}^{x}(\omega),-\bar{t}\right)$ : the first one goes to $(x, 0)$ and the other, which we denote by $\gamma_{1}$, goes to $(w, 0)$, for some $w \geq y$.

Now, for each $t_{n_{k}}>\bar{t}$, let us take a path $\left(x_{0}, s_{0}\right),\left(x_{1}, s_{1}\right), \ldots,\left(x_{m}, s_{m}\right)$ with $\left(x_{0}, s_{0}\right)=$ $\left(y_{n_{k}},-t_{n_{k}}\right)$ and $\left(x_{m}, s_{m}\right)=(x, 0)$. We have $-\bar{t} \in\left[s_{i}, s_{i+1}\right)$ for some $i>1$, therefore, since $\gamma_{2}=\left(x_{i},-\bar{t}\right),\left(x_{i+1}, s_{i+1}\right) \ldots,\left(x_{m}, s_{m}\right)$ gives a path from $\left(x_{i},-\bar{t}\right)$ to $(x, 0)$ and we have necessarily $x_{i} \geq \hat{l}_{\bar{t}}^{x}(\omega), \gamma_{2}$ has to cross $\gamma_{1}$. Therefore there is a path from $\left(x_{i},-\bar{t}\right)$ to $(w, 0)$ and so there is one path from $\left(x_{0}, s_{0}\right)=\left(y_{n_{k}},-t_{n_{k}}\right)$ to $(w, 0)$. We have proved that, for each $y>x$ there exists $w \geq y$ such that, for all $k$ sufficiently big we have $\varphi\left(t_{n_{k}}, \theta_{-t_{n_{k}}} \omega\right) \eta^{y_{n_{k}}}(w)=1$. This means that $\sup \{x: \zeta(x)=1\}=+\infty$ and the proof is complete.

Remark 5 . Note that, from properties (4.10), (4.12) and (4.14) follows easily that, for $\mathbb{P}$-almost all $\omega, \lim _{t \rightarrow+\infty} \varphi\left(t, \theta_{-t}\right) \eta^{-}=\sigma^{+}(\omega)$ and the same result holds for the pullback trajectory of $\eta^{+}$. Clearly, this property can be extended to all the "one-sided" configurations, i.e., configurations of the type $\eta^{B}$, where $B=(-\infty, x]$ or $B=[x,+\infty)$. This shows that, if $\widetilde{\mathcal{B}}$ is the collection of all the one-sided configurations of $X$, then the random set $\left\{\sigma^{+}\right\}$is a $\widetilde{\mathcal{B}}$-random attractor for $\varphi$ and so, in this case $\Omega_{X}$ is not the minimal attractor for $\widetilde{\mathcal{B}}$.

Note also that, as can be seen in the proof of the proposition above, if $\omega$ is such that (4.16) does not hold, $\Omega_{X}(\omega)$, besides $\overline{0}$ and $\sigma^{+}(\omega)$, can contain only configurations of the form $\sigma^{+}(\omega) \wedge \eta^{(-\infty, x]}$ and $\sigma^{+}(\omega) \wedge \eta^{[x,+\infty)}$ for $x \in Z$. 
Acknowledgment. The authors are deeply indebted to Prof. F. Flandoli, for his suggestions and encouragement.

\section{References}

[A] L. Arnold, Random Dynamical Systems. Springer, Berlin Heidelberg New York, 1998.

[AC] L. Arnold, I. Chueshov, Order preserving random dynamical systems: equilibria, attractors, applications, Dynamics and Stability of Systems 13 (265-280), 1998.

[Br] P. Brémaud, Markov Chains, Gibbs Fields, Monte Carlo Simulation and Queues. Texts in applied Math.31, Springer Verlag, New York 1999.

[C] H. Crauel, Markov Measures for Random Dynamical Systems, Stochastics and Stoch. Reports 37 (153-173), 1991.

[C2] H. Crauel, Global random attractors are uniquely determined by attracting deterministic compact sets, Annali di Matematica pura ed applicata IV, Vol. 176 (57-72), 1999

[CF] H. Crauel, F. Flandoli, Attractors for Random Dynamical Systems, Probability Theory and Related Fields 100 (365-393), 1994.

[Ch] I. Chueshov, Monótone Random Systems. Theory and Applications. Lecture Notes in Mathematics 1779, Springer-Verlag, Berlin 2002.

[CS] I. Chueshov, M. Scheutzow, On the structure of attractors and invariant measures for a class of monotone random systems, Dynamical Systems 19 (127-144), 2004

[CV] C. Castaing, M. Valadier, Convex Analysis and Measurable Multifunctions. Lecture Notes in Mathematics 580, Springer, Berlin Heidelberg New York, 1977.

[Du] R. Durrett, Oriented percolation in two dimensions, Ann. Probab. 12 (999-1040), 1984.

[EK] S. Ethier, T.G. Kurtz, Markov Processes. Characterization and Convergence. Wiley, New York, 1986.

[FG] P. Ferrari, A. Galves, Construction of Stochastic Processes, Coupling and Regeneration. 2003, to appear.

[Fi] J.A. Fill, An interruptible algorithm for Perfect Sampling via Markov Chains, The Annals of Applied Probability Vol.8 No.1 (131-162), 1998.

[HP] S. Hu, N.S. Papageorgiou. Handbook of Multivalued Analysis, vol 1: Theory. Kluver Academic Publishers, Dordrecht 1997. 
[Mi] I. Minelli, Patwise convergence to equilibrium of the supercritical Contact Process, Ph. D. Thesis, 2004.

[K] Y. Kifer, Ergodic Theory of Random Transformations. Birkhäuser, Boston Basel Stuttgart, 1986.

[Li1] T.M. Liggett, Interacting Particle Systems, Springer Verlag, New York 1985.

[Li2] T.M. Liggett, Stochastic Interacting Systems: Contact, Voter and Exclusion Processes, Grundlehren der Mathematischen Wissenschaften, Springer-Verlag, Berlin 1999.

[PW] G. Propp, D.B. Wilson, Exact sampling with coupled Markov Chains and applications to Statistical Mechanics, Random Structures Algorithms 9 (1996), no.1-2, 223-252. 